PROJECT INFANS - DQ45420

Main Questionnaire - NEW VERSION

\begin{tabular}{|c|c|c|c|c|c|c|c|c|c|}
\hline \multirow{2}{*}{ CENTER } & Delhi & 01 & Kolkata & 02 & Mumbai & 03 & Chennai & 04 \\
\cline { 2 - 8 } & Lucknow & 05 & Patna & 06 & Pune & 07 & Vizag & 08 \\
\cline { 2 - 8 } & Jalandhar & 09 & Bhubaneshwar & 10 & Bhilai & 11 & Cochin & 12 \\
\hline
\end{tabular}

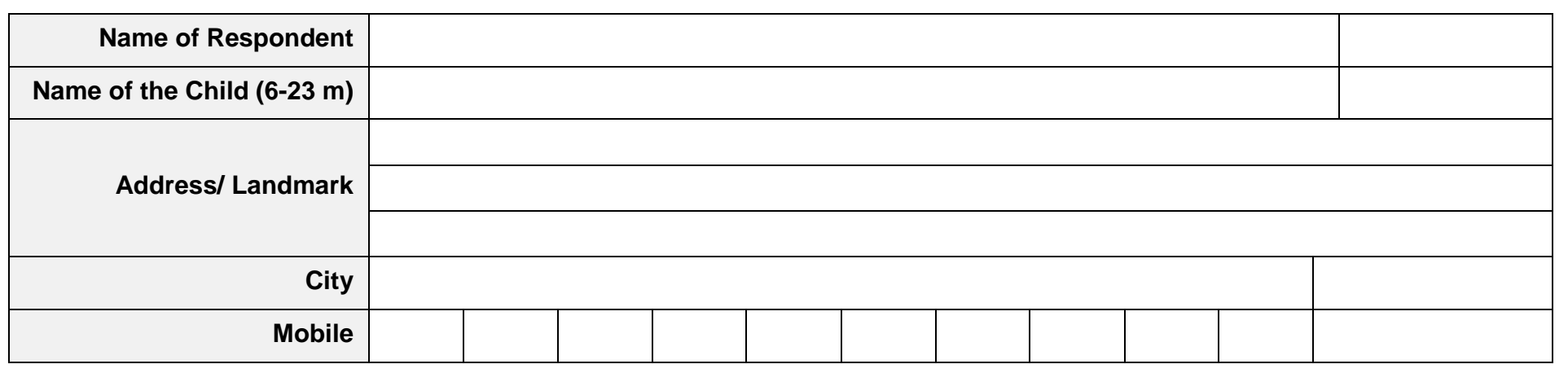

\begin{tabular}{|c|c|c|c|c|c|c|c|}
\hline \multicolumn{6}{|l|}{ Name of Supervisor: } & Supervisor's Code & $713-714$ \\
\hline \multicolumn{6}{|l|}{ Interviewer's Name: } & Interviewer's Code & $715-716$ \\
\hline \multicolumn{6}{|l|}{ Starting Area: } & SA Code & $1888-89$ \\
\hline Interview Number: & & & & \multicolumn{2}{|c|}{$1890-92$} & & \\
\hline Date of Interview & 2 & 0 & 1 & 3 & $\begin{array}{l}717- \\
720\end{array}$ & & \\
\hline
\end{tabular}

\begin{tabular}{|c|c|c|c|c|c|c|c|c|c|}
\hline \multicolumn{3}{|c|}{ ACCOMPANIED } & \multicolumn{4}{|c|}{ BACK CHECKED } & \multicolumn{3}{|c|}{ SCRUTINIZED } \\
\hline & & & & $\mathbf{P}$ & $\mathbf{T}$ & & & & \\
\hline TL & 1 & & TL & 1 & 5 & & TL & 1 & \\
\hline EIC & 2 & & EIC & 2 & 6 & & EIC & 2 & \\
\hline OFE & 3 & & OFE & 3 & 7 & & OFE & 3 & \\
\hline \multirow[t]{2}{*}{ FM } & 4 & & FM & 4 & 8 & & FM & 4 & \\
\hline & & $721-724$ & & & & $725-732$ & & & $733-736$ \\
\hline \multicolumn{3}{|c|}{ Signature: TL/EIC/OFE/FI } & \multicolumn{4}{|c|}{ Signature: TL/EIC/OFE/FM } & \multicolumn{3}{|c|}{ Signature: TL/EIC/OFE/FM } \\
\hline
\end{tabular}

\begin{tabular}{|c|c|c|c|c|c|}
\hline \multicolumn{6}{|c|}{ QUOTA GRID } \\
\hline AGE OF CHILD & \multicolumn{2}{c|}{ SEC } & \multicolumn{2}{c|}{ SEGMENT } \\
\hline 6-12 months & 1 & A & 1 & Current Buyer & 1 \\
\hline 13-18 months & 2 & B & 2 & Non Buyer & 2 \\
\hline 19- 23 months & 3 & C & 3 & & \\
\hline & & D & 4 & & \\
\hline & & E & 5 & & 739 \\
\hline
\end{tabular}

TIME OF START:

TIME OF END: TOTAL DURATION: 


\section{Main Questionnaire}

\section{Purchase \& Consumption Section}

\section{CODE RESPONSES FOR Q301- Q303 IN THE GRID GIVEN AFTER Q303}

\section{SHOW PHOTOCARDS}

Q301 Please tell me in past 3 days what all has [REFERENT CHILD] consumed? Please include all food items and beverages the child has consumed. MULTIPLE CODING POSSIBLE

कृप्या मुझे बताए पिछले 3 दिनो मे $\ldots . . . \ldots \ldots . . .$. ने क्या सब खाया था ? कृप्या सभी खाद्य वस्तुओं और पेय पदार्थो को शामिल करे जो कि बच्चे ने खायी/ पी है।

Please do not include today's day when we say past 3 days. Please include 3 days with third day ending yesterday night - 12am

कृप्या आज का दिन न मिलाएं। पिछले 3 दिनो के बारे मे बताए जो कल रात 12 बजे खतम हुए हों ।

\section{SHOW PHOTOCARD FOR EACH CATEGORY CODED IN Q301}

Q303 SHOWCARD Q303 Please tell me approx. how many servings of each item does the child eat in a week? SINGLE CODING ONLY

कृप्या मुझे बताए अंदाजन एक सप्ताह मे बच्चा प्रत्येक वस्तु की कितनी सर्विग खाता है ?

\begin{tabular}{|c|c|c|}
\hline \multicolumn{3}{|c|}{ SHOW CARD Q303 } \\
\hline 6 times per day or more & प्रति दिन 6 या ज्यादा बार & 01 \\
\hline 5 times per day & प्रति दिन 5 बार & 02 \\
\hline 4 times per day & प्रति दिन 4 बार & 03 \\
\hline 3 times per day & प्रति दिन 3 बार & 04 \\
\hline 2 times per day & प्रति दिन 2 बार & 05 \\
\hline 1 time per day & प्रति दिन 1 बार & 06 \\
\hline 5-6 times per week & प्रति सप्ताह 5-6 बार & 07 \\
\hline 3-4 times per week & प्रति सप्ताह $3-4$ बार & 08 \\
\hline 1-2 times per week & प्रति सप्ताह $1-2$ बार & 09 \\
\hline Less often & कम बार & 10 \\
\hline During emergencies/ special occasions & $\begin{array}{l}\text { आपातस्थितियों/ विशेष अवसरो के } \\
\text { दौरान }\end{array}$ & 11 \\
\hline Don't know / remember & पता नही/ याद नही & 98 \\
\hline
\end{tabular}




\begin{tabular}{|c|c|c|c|}
\hline \multicolumn{3}{|c|}{ Q301 } & \multirow{2}{*}{$\begin{array}{c}\text { Q303 } \\
\text { Servings }\end{array}$} \\
\hline \multicolumn{2}{|c|}{ Name of Item Consumed in Past 3 Days } & \multirow{2}{*}{$\begin{array}{c}\text { Item Code } \\
084\end{array}$} & \\
\hline Breast Milk & माँ का दूध & & \\
\hline $\begin{array}{l}\text { Cereals like Daliya/ Khichdi/ Mixed } \\
\text { Grains/ Sewiya }\end{array}$ & $\begin{array}{l}\text { सीरियल्स जैसे दलिया/खिचड़ी/मिले } \\
\text { अनाज/सेवईंया }\end{array}$ & 151 & \\
\hline $\begin{array}{l}\text { Cereals like Ragi/ Barleyl Bajral } \\
\text { Maize/ Corn }\end{array}$ & सीरियल्स जैसे रागी/जों/बाजरा/मक्का/दाना & 152 & \\
\hline Oats/ Corn flakes/ Rice flakes & ओट्स/ कोर्नफ्लेक्स/ चावल के फ्लेक्स & 153 & \\
\hline Rice/ Rice based dishes & चावल/ चावल से बने व्यंजन & 154 & \\
\hline Packaged Infant Cereals & पैकेज्ड इनफेंट सीरियल्स/ बेबी फूड & 155 & \\
\hline Legumes like pulses, chana etc. & फली जैसे दालें, चना आदि & 156 & \\
\hline $\begin{array}{l}\text { Roti, Paratha, Bread, Dosa, Poori } \\
\text { etc. }\end{array}$ & रोटी, पराठा, ब्रेड, डोसा, पूरी आदि & 157 & \\
\hline Packaged Powdered Milk & पैकेज्ड पाउडर्ड दूध & 085 & \\
\hline $\begin{array}{l}\text { Homemade Snacks like poha, } \\
\text { semolina/ suji/ rawa, halwa, upma, idli, } \\
\text { puffed rice, Idiyappam, sattu, chattuah, } \\
\text { sabudana etc. }\end{array}$ & $\begin{array}{l}\text { घर कें बने स्नेक्स जैसे पोहा, } \\
\text { सेमोलिना/सूजी/रवा, हलवा, उपमा, इडली, } \\
\text { फूले चावल, इडियापन, सत्तू, छतूआ, } \\
\text { साबुदाना आदि }\end{array}$ & 158 & \\
\hline $\begin{array}{l}\text { Cow milk/ buffalo milk (packaged or } \\
\text { loose) }\end{array}$ & $\begin{array}{l}\text { गाय का दूध/भैंस का दूध (पैकेज्ड या } \\
\text { खुला) }\end{array}$ & 159 & \\
\hline $\begin{array}{l}\text { Milk products like curd, raita, lassi/ } \\
\text { chaach, cream, tea, coffee, paneer, } \\
\text { khoya, shakes, ice cream etc }\end{array}$ & $\begin{array}{l}\text { दूध के प्रोडक्ट्स जैसे दही, रायता, } \\
\text { लस्सी/छाछ, क्रीम, चाय, कॉफी, पनीर, } \\
\text { खोवा, शेक्स, आईसक्रीम आदि. }\end{array}$ & 160 & \\
\hline $\begin{array}{l}\text { Non vegetarian foods and non veg } \\
\text { soups }\end{array}$ & माँसाहारी खाने और माँसाहारी सूप्स & 161 & \\
\hline Infant formulas & इनफेंट फार्मूला & 162 & \\
\hline Fruits/ Fruit Juices/ Neembu pani & फल/ फलों के जूस/ नींबू पानी & 163 & \\
\hline $\begin{array}{l}\text { Packaged snacks/ foods like biscuits, } \\
\text { chips, soft drinks, confectionary items, } \\
\text { namkeens, milk additives likes Horlicks } \\
\text { etc. }\end{array}$ & $\begin{array}{l}\text { पैकेज्ड स्नेक्स/फूड्स जैसे बिस्कुट्स, चिप्स, } \\
\text { सॉफ्ट ड्रिंक्स, कॉन्फेक्शनरी साम्रियाँ, } \\
\text { नमकीरींस, दूध एडीटिव्स जैसे हॉरलिक्स } \\
\text { आदि }\end{array}$ & 164 & \\
\hline Vegetables and vegetable soups & सब्जियाँ और सब्जियों के सूप्स & 165 & \\
\hline \multicolumn{4}{|l|}{$\begin{array}{l}\text { Others (PLEASE SPECIFY) } \\
\text { अन्य (कृप्या स्पष्ट करें) }\end{array}$} \\
\hline \multicolumn{4}{|l|}{$\begin{array}{l}\text { Others (PLEASE SPECIFY) } \\
\text { अन्य (कृप्या स्पष्ट करें) }\end{array}$} \\
\hline \multicolumn{4}{|l|}{$\begin{array}{l}\text { Others (PLEASE SPECIFY) } \\
\text { अन्य (कृप्या स्पष्ट करें) }\end{array}$} \\
\hline \multicolumn{4}{|l|}{$\begin{array}{l}\text { Others (PLEASE SPECIFY) } \\
\text { अन्य (कृप्या स्पष्ट करें) }\end{array}$} \\
\hline \multicolumn{4}{|l|}{$\begin{array}{l}\text { OtherS (PLEASE SPECIFY) } \\
\text { अन्य (कृप्या स्पष्ट करें) }\end{array}$} \\
\hline \multicolumn{4}{|l|}{$\begin{array}{l}\text { Others (PLEASE SPECIFY) } \\
\text { अन्य (कृप्या स्पष्ट करें) }\end{array}$} \\
\hline \multicolumn{4}{|l|}{$\begin{array}{l}\text { Others (PLEASE SPECIFY) } \\
\text { अन्य (कृप्या स्पष्ट करें) }\end{array}$} \\
\hline \multicolumn{4}{|l|}{$\begin{array}{l}\text { Others (PLEASE SPECIFY) } \\
\text { अन्य (कृप्या स्पष्ट करें) }\end{array}$} \\
\hline \multicolumn{4}{|l|}{$\begin{array}{l}\text { OtherS (PLEASE SPECIFY) } \\
\text { अन्य (कृप्या स्पष्ट करें) }\end{array}$} \\
\hline $\begin{array}{l}\text { Others (PLEASE SPECIFY) } \\
\text { अन्य (कृप्या स्पष्ट करें) }\end{array}$ & & & \\
\hline
\end{tabular}

\begin{tabular}{|c|c|c|c|c|}
\hline \multicolumn{5}{|c|}{ CLASSIFY AS: } \\
\hline $\begin{array}{r}\text { If [REF CHILD] has consumed packaged } \\
\text { infant cereal in last } 3 \text { days }\end{array}$ & Coded 155 in Q301 & CURRENT BUYER & 1 & GO TO Q304 \\
\hline Else & $\begin{array}{l}\text { NOT coded } 155 \text { in } \\
\text { Q301 }\end{array}$ & NON BUYER & 2 & GO TO Q316 \\
\hline
\end{tabular}


ASK Q304-Q315 IF PACKAGED INFANT CEREAL (155) CODED IN Q301

CODE RESPONSES FOR Q304- Q307 IN THE GRID GIVEN AFTER Q307

Q304 What was the brand of packaged infant cereal/ baby foods that was given to [REFERENT CHILD]? RECORD VERBATIM

को दिए गए पैकेज्ड शिशु अनाज/ शिशु आहार का ब्रांड क्या था ?

Q305 SHOWCARD Q305 Please look at this card and tell me in which pack type did you buy (MENTION BRANDS CODED IN Q304 ONE BY ONE)? SINGLE CODING ONLY FOR EACH BRAND कृप्या इस कार्ड को देखे और मुझे बताए आपने को किस पैक प्रकार मे खरीदा था ?

\begin{tabular}{|r|r|l|}
\hline \multicolumn{3}{|c|}{ SHOW CARD Q305 } \\
\hline Poly-pack & पॉली पैक & 01 \\
\hline Can & कैन & 02 \\
\hline Tin & टिन & 03 \\
\hline Plastic pack & प्लास्टिक पैक & 04 \\
\hline Tetra pack & टैट्रा पैक & 05 \\
\hline Paper pack & पैपर पैक & 06 \\
\hline Sachet & सैशे & 07 \\
\hline PET bottle & पैट बोतल & 08 \\
\hline Glass bottle & ग्लास की बोतल & 09 \\
\hline Bagging Box & वैगिंग बॉक्स & 10 \\
\hline
\end{tabular}

Q306 And what size did you buy of और आपने

(MENTION BRANDS CODED IN Q304 ONE BY ONE)? का क्या साइज खरीदा ?

PLEASE ASK RESPONDENT TO SHOW THE PACK IF AVAILABLE AT HOME AND NOTE PACK SIZE FROM THE PACK.

\section{IF PACK NOT AVAILABLE OR RESPONDENT REFUSED TO SHOW THE PACK, RECORD}

PLEASE RECORD THE PACK SIZE IN GRAMS. RECORD VERBATIM WITH LEADING ZEROES

Q307 a) Now please tell me out of

(MENTION BRANDS CODED IN Q304 ONE BY ONE) which cereal/ baby food does [REFERENT CHILD] consume most? SINGLE CODING ONLY

अब कृप्या मुझे बताए ............ मे से कौन सा अनाज/ शिशु आहार

ज्यादातर खाता/पीता है ?

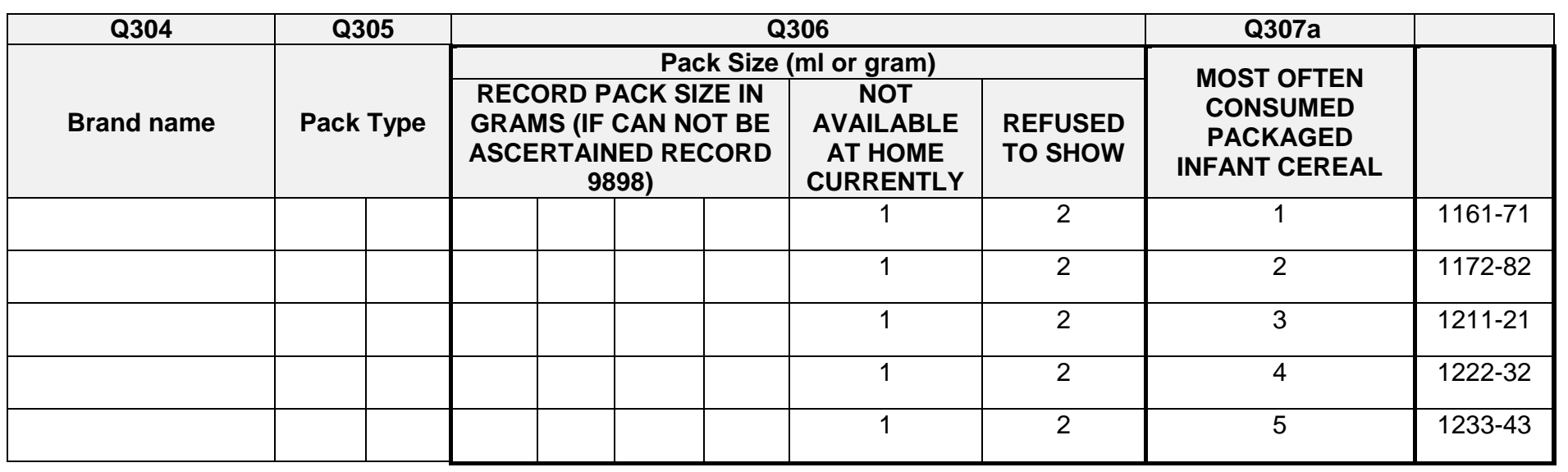

Q307 b) When was the last time when you served packaged infant cereals to

(REFERENT CHILD)?

SINGLE CODING ONLY

पिछली बार कब आपने

के लिए पैकेज्ड शिशु अनाज को सर्व किया था ?

\begin{tabular}{|r|c|c|}
\hline Same day & उसी दिन & 1 \\
\hline Yesterday & कल & 2 \\
\hline 2 days back & 2 दिन पहले & 3 \\
\hline 3 days back & 3 दिन पहले & 4 \\
\hline
\end{tabular}

INSTRUCTION TO INTERVIEWER: PLEASE ASK RESPONDENT TO BRING THE SPOON THEY NORMALLY USE TO POUR PACKAGED INFANT CEREAL WHILE PREPARING FOR CHILD

ALSO, TAKE OUT THE SEMOLINA PACKET 
I have with me some semolina. Please tell me with the help of this spoon, how much quantity of packaged infant cereal you gave to___ (REFERENT CHILD) during this occasion?

मेरे पास कूछ सेमोलिना/ सूजी है। कृप्या मुझे इस चम्मच की मदद से बताए, पैकेज्ड शिशु अनाज की कितनी मात्रा आप ने इस अवसर के दौरान ................ को दी ?

\section{ASK THE RESPONDENT TO PUT THE SEMOLINA IN SPOON. MEASURE THE QUANTITY USING STANDARD} BEAKER AND RECORD BELOW

\begin{tabular}{|l|l|l|l|l|l|l} 
QUANTITY LEVEL & & & \\
1246-48
\end{tabular}

SAY: Now, I'll ask you some questions about the packaged infant cereal/ baby food that is consumed by the child most often i.e. (MENTION CEREAL NAME CODED IN Q307)

कहे: अब, मै आपसे पैकेज्ड शिशु अनाज/ शिशु आहार के बारे मे कूछ प्रश्न पूछूंगा जो कि बच्चे द्वारा ज्यादातर खाया जाता है मतलब .................।

Q309 SHOWCARD Q309 Please tell me approx. how many servings does the child eat of this packaged infant cereal/ baby food in a week? SINGLE CODING ONLY

कृप्या मुझे बताए अंदाजन एक सप्ताह मे इस पैकेज्ड शिशु अनाज/ शिशु आहार की कितनी सर्विग बच्चा खाता है ?

\begin{tabular}{|r|r|c|}
\hline 6 times per day or more & प्रति दिन 6 या ज्यादा बार & 01 \\
\hline 5 times per day & प्रति दिन 5 बार & 02 \\
\hline 4 times per day & प्रति दिन 4 बार & 03 \\
\hline 3 times per day & प्रति दिन 3 बार & 04 \\
\hline 2 times per day & प्रति दिन 2 बार & 05 \\
\hline 1 time per day & प्रति दिन 1 बार & 06 \\
\hline 5-6 times per week & प्रति सप्ताह 5-6 बार & 07 \\
\hline 3-4 times per week & प्रति सप्ताह 3-4 बार & 08 \\
\hline 1-2 times per week & प्रति सप्ताह 1-2 बार & 09 \\
\hline Less often & कापर्थितियों / विशेष अवसरो के & 10 \\
\hline
\end{tabular}

INSTRUCTION TO INTERVIEWER: NOW AGAIN TAKE THE SPOON THE RESPONDENT NORMALLY USES TO POUR PACKAGED INFANT CEREAL WHILE PREPARING FOR CHILD

ALSO, TAKE OUT THE SEMOLINA PACKET

Q310 Now please tell me with the help of this spoon, how much quantity of packaged infant cereal you generally use while making one serving for the child.

अब कृप्या इस चम्मच की मदद से मुझे बताए, पैकेज्ड शिशु अनाज की कितनी मात्रा आप सामान्यता बच्चे के लिए एक सर्विग बनाते समय उपयोग करते है।

\section{ASK THE RESPONDENT TO PUT THE SEMOLINA IN SPOON. MEASURE THE QUANTITY USING STANDARD} BEAKER AND RECORD BELOW

$$
\text { QUANTITY LEVEL }
$$

$1251-53$

Q311 SHOWCARD Q311 Please look at this card and tell me which of the following applies to the way this packaged infant cereal/ baby food is generally prepared? SINGLE CODING ONLY कृप्या इस कार्ड को देखे और मुझे बताए निम्नलिखित मे से कौन सा इस पैकेज्ड शिशु अनाज/ शिशु आहार को बनाने के तरीके पर लागु होता है ?

\begin{tabular}{|c|c|c|}
\hline Mixed with normal water & $\begin{array}{l}\text { सामान्य पानी के साथ } \\
\text { मिलाकर }\end{array}$ & 01 \\
\hline Mixed with warm water & $\begin{array}{r}\text { गुनगुने पानी के साथ } \\
\text { मिलाकर }\end{array}$ & 02 \\
\hline Mixed with hot water & $\begin{array}{l}\text { गर्म पानी के साथ } \\
\text { मिलाकर }\end{array}$ & 03 \\
\hline Mixed with normal milk & $\begin{array}{l}\text { सामान्य दूध के साथ } \\
\text { मिलाकर }\end{array}$ & 04 \\
\hline Mixed with warm milk & $\begin{array}{l}\text { गुनगुने दूध के साथ } \\
\text { मिलाकर }\end{array}$ & 05 \\
\hline Mixed with hot milk & गर्म दूध के साथ मिलाकर & 06 \\
\hline Any other (please specify) & $\begin{array}{r}\text { कोई अन्य (कृप्या स्पष्ट } \\
\text { करे) }\end{array}$ & \\
\hline
\end{tabular}

Q312 Please look at this card and tell me, SHOWCARD Q312 how long back did you buy this packaged infant cereal/ baby food? SINGLE CODING ONLY

कृप्या इस कार्ड को देखे और मुझे बताए, कितने समय पहले आपने इस पैकेज्ड शिशु अनाज/ शिशु आहार को खरीदा था ? 


\begin{tabular}{|c|c|c|}
\hline In last 1 week & पिछले 1 सप्ताह मे & 1 \\
\hline In last 2 weeks but not in last 1 week & $\begin{array}{r}\text { पिछले } 2 \text { सप्ताह मे परन्तु पिछले } 1 \\
\text { सप्ताह मे नही }\end{array}$ & 2 \\
\hline In last 1 month but not in last 2 weeks & $\begin{array}{r}\text { पिछले } 1 \text { महीने मे परन्तु पिछले } 2 \\
\text { सप्ताह मे नही }\end{array}$ & 3 \\
\hline In last 2 months but not in last 1 month & $\begin{array}{r}\text { पिछले } 2 \text { महीनो मे परन्तु पिछले } 1 \\
\text { महीनो मे नही }\end{array}$ & 4 \\
\hline In last 6 months but not in last 2 months & $\begin{array}{r}\text { पिछले } 6 \text { महीनो मे परन्तु पिछले } 2 \\
\text { महीनो मे नही }\end{array}$ & 5 \\
\hline Before last 6 months & पिछले 6 महीनो से पहले & 6 \\
\hline
\end{tabular}

Q329 Please look at this card and tell me, SHOWCARD Q329, from which place do you purchase this packaged infant cereal/ baby food most often? MULTIPLE CODING POSSIBLE

कृपया इस कार्ड को देखें और मुझे बताये कि आप किन जगहों से इस पैकेज्ड इनफेंट सीरियल/बेबी फूड को ज्यादातर खरीदती हैं ?

\begin{tabular}{|r|r|c|}
\hline \multicolumn{2}{|c|}{ Q329 } \\
\hline Kirana store near the locality & $\begin{array}{r}\text { क्षेत्र के निकट किराना स्टोर } \\
\text { Grocery store/ General merchant }\end{array}$ & 01 \\
\hline Modern format outlet & आधुनिक स्टोर/ जनरल मर्चेट & 02 \\
\hline Pharmacy/ Chemist shop & फार्मसी/ कैमिस्टि शॉप & 04 \\
\hline From doctor & डॉक्टर से & 05 \\
\hline Street vendors & स्ट्रीट वेंडर्स & 06 \\
\hline Dairy/ Milk Outlet & डेयरी/ दूध की दूकान & 07 \\
\hline Bakery & अेकरी & 09 \\
\hline Any other (please specify) & कोई अन्य (कृप्या स्पष्ट करे) & \\
\hline Any other (please specify) & कोई अन्य (कृप्या स्पष्ट करे) & \\
\hline
\end{tabular}

Q313 SHOWCARD Q313 Please tell me why do you serve packaged infant cereal/ baby food to [REFERENT CHILD]? MULTIPLE CODING POSSIBLE

कृप्या मुझे बताए आप .............. के लिए पैकेज्ड शिशु अनाज/ शिशु आहार क्यों सर्व करते है ?

\begin{tabular}{|c|c|c|}
\hline They are healthy for child & यह बच्चो के लिए स्वास्थ्यदायक है & 01 \\
\hline $\begin{array}{r}\text { The doctor/ health professional } \\
\text { recommended this }\end{array}$ & डॉक्टर/ स्वास्थ्य पेशेवर ने इसकी सिफारिश की थी & 02 \\
\hline $\begin{array}{r}\text { My child likes the taste of packaged infant } \\
\text { cereal/ baby foods }\end{array}$ & $\begin{array}{r}\text { मेरा बच्चा पैकेज्ड शिशु अनाज/ शिशु आहार के स्वाद को } \\
\text { पसंद करता है }\end{array}$ & 03 \\
\hline $\begin{array}{r}\text { My child gets digestive problem after eating } \\
\text { other foods }\end{array}$ & $\begin{array}{l}\text { अन्य खाने के बाद मेरे बच्चे को पाचन की परेशानी हो } \\
\text { जाती है }\end{array}$ & 04 \\
\hline My child is too young for other foods & मेरा बच्चा अन्य आहार के लिए बहुत छोटा है & 05 \\
\hline My child is too old for only milk & केवल दूध के लिए मेरा बच्चा बहुत बडा है & 06 \\
\hline I don't prefer homemade cereals & मै होममेड सीरियल को पसंद नही करती & 07 \\
\hline $\begin{array}{r}\text { I don't have time to prepare homemade } \\
\text { cereals }\end{array}$ & $\begin{array}{r}\text { मेरे पास बच्चे के लिए घर पर बना अनाज बनाने का } \\
\text { समय नही है }\end{array}$ & 08 \\
\hline Any other (please specify) & कोई अन्य (कृप्या स्पष्ट करे) & \\
\hline Any other (please specify) & कोई अन्य (कृप्या स्पष्ट करे) & \\
\hline
\end{tabular}


Please look at this card and tell me, SHOWCARD Q314 who else in your household consumes the packaged infant cereal/ baby food that is prepared for the kids 6-23 months old. MULTIPLE CODING POSSIBLE

कृप्या इस कार्ड को देखे और मुझे बताए, आपके परिवार मे और कौन पैकेज्ड शिशु अनाज/ शिशु आहार को खाता/ पीता है जो कि 6-23 महीने की उम्र के लिए बनाया जाता है।

\begin{tabular}{|r|r|c|}
\hline Kids between 2-5 years & $2-5$ वर्ष के बीच के बच्चे & 1 \\
\hline Kids between 6-12 years & $6-12$ वर्ष के बीच के बच्चे & 2 \\
\hline Kids between 13-17 years & $13-17$ वर्ष के बीच के बच्चे & 3 \\
\hline Adults between 18-25 years & $18-25$ वर्ष के बीच के व्यस्क & 4 \\
\hline Adults between 26-40 years & $26-40$ वर्ष के बीच के व्यस्क & 5 \\
\hline Adults above 40 years & 40 वर्ष से बडे के व्यस्क & 6 \\
\hline $\begin{array}{r}\text { No one else consumes packaged } \\
\text { infant cereal/ baby food prepared } \\
\text { for kids 6-23 months old }\end{array}$ & पैकेज्ड शिशु अनाज/ शिशु आहार को कोई & 9 \\
और नही खाता पीता & \\
\hline
\end{tabular}

Q315 Thinking about the total packaged infant cereal/ baby food consumed in your household in last 1 week, please divide it among members in your household who consume packaged infant cereal/ baby food as per their share in total quantity consumed. RECORD VERBATIM FOR EACH MEMBER CODED IN Q314. RECORD VERBATIM FOR THE SELECTED CHILD. RECORD WITH LEADING ZEROES

पिछले 1 सप्ताह मे आपके परिवार मे खाए गए कूल पैकेज्ड शिशु अनाज/ शिशु आहार के बारे मे सोचते हुए, कृप्या इस आपके परिवार मे सदस्यों के बीच जो पैकेज्ड शिशु अनाज/ शिशु आहार को खाते है खपत की गयी कूल मात्रा मे उनके हिस्से के अनुसार बांटे।

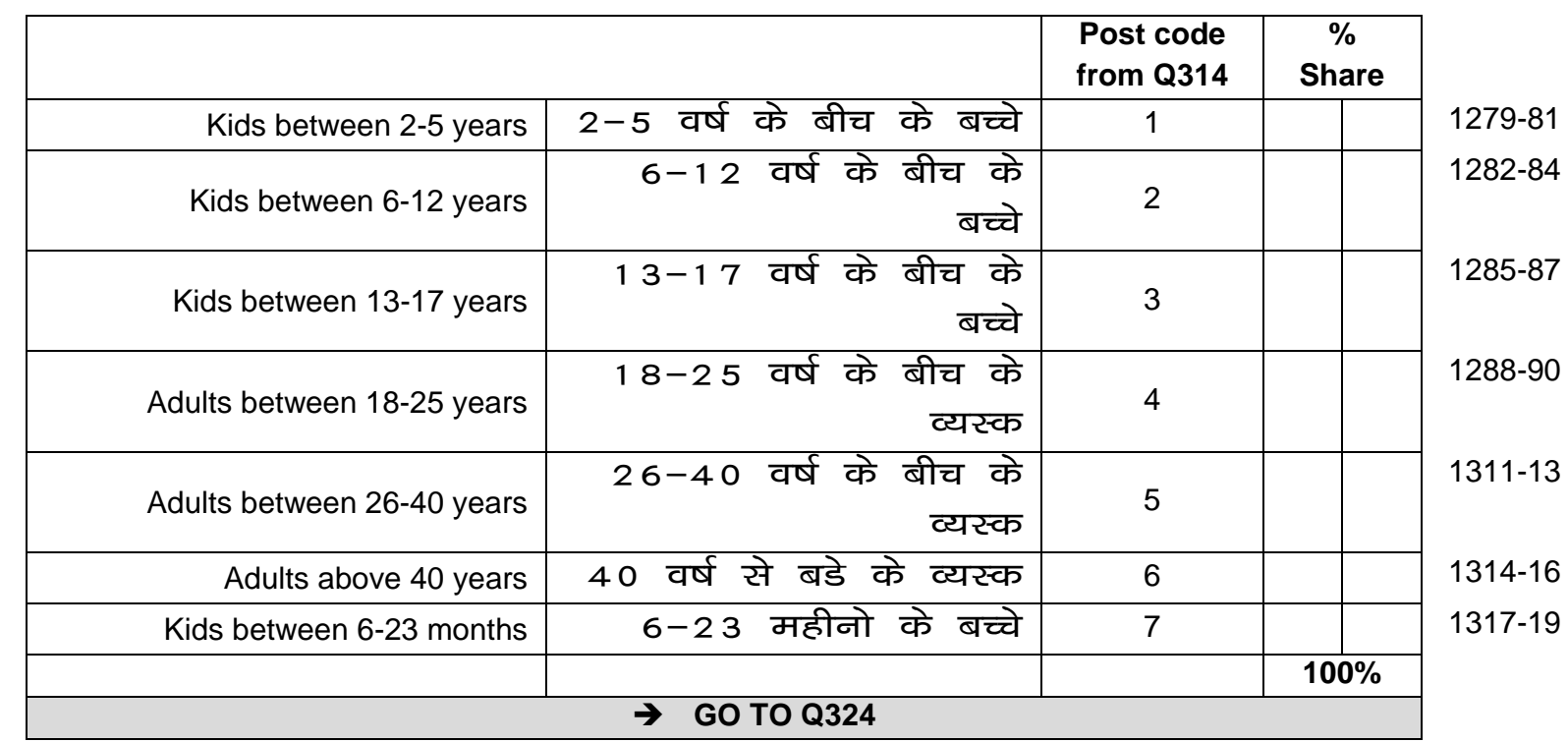

\section{ASK Q316-Q320 IF PACKAGED INFANT CEREAL (155) NOT CODED IN Q301 (PACKAGED INFANT CEREAL NOT} SERVED IN PAST 3 DAYS)

\section{CODE RESPONSES FOR Q316- Q317 IN THE GRID GIVEN AFTER Q317}

Q316 DO NOT EXPOSE THE LIST Please tell me which brands of packaged infant cereals/ baby foods are you aware of? MULTIPLE CODING POSSIBLE

कृप्या मुझे बताए पैकेज्ड शिशु अनाज/ शिशु आहार के किन ब्रांडस से आप अवगत है ?

Q317 At what price is a pack of packaged infant cereal/ baby food is available in market? Please specify the pack size as well (in gm). RECORD VERBATIM WITH LEADING ZEROES

पैकेज्ड शिशु अनाज/ शिशु आहार का पैक बाजार मे किस कीमत पर उपलब्ध है ? कृप्या पैक साइज को भी स्पष्ट करे।

\begin{tabular}{|c|c|c|c|c|}
\hline \multirow{2}{*}{\multicolumn{3}{|c|}{\begin{tabular}{|c|} 
Q316 \\
DO NOT EXPOSE THE LIST/ DO NOT PROMPT
\end{tabular}}} & \multirow{2}{*}{$\begin{array}{c}\text { Q317.1 } \\
\text { PRICE (in Rs.) }\end{array}$} & \multirow{2}{*}{$\begin{array}{c}\text { Q317.2 } \\
\text { PACK SIZE (in gm) }\end{array}$} \\
\hline & & & & \\
\hline Cerelac & सेरेलक & 01 & & \\
\hline Nestum & नेस्टम & 02 & & \\
\hline Farex & फैरेक्स & 03 & & \\
\hline Gerber & जरबर & 04 & & \\
\hline Any other & कोई अन्य & & & \\
\hline Any other & कोई अन्य & & & \\
\hline Any other & कोई अन्य & & & \\
\hline Not aware/ Cannot recall & $\begin{array}{l}\text { अवगत नही/ याद } \\
\text { नही }\end{array}$ & 98 & & \\
\hline
\end{tabular}

$1320-27$

Q318 Have you ever served packaged infant cereals/ baby foods to [REFERENT CHILD]? SINGLE CODING ONLY क्या आपने कभी ............... को पैकेज्ड शिशु अनाज/ शिशु आहार को सर्व किया है ? 


\begin{tabular}{|l|c|l|}
\hline $\begin{array}{l}\text { Yes } \\
\text { हाँ }\end{array}$ & 1 & $\rightarrow$ GO TO Q321 \\
\hline $\begin{array}{l}\text { No } \\
\text { नही }\end{array}$ & 2 & $\rightarrow$ GO TO Q319 \\
\hline
\end{tabular}

\section{ASK Q319/ Q320 IF '2' CODED IN Q318}

Q319 SHOWCARD Q319 You said that you never served packaged infant cereals/ baby foods to [REFERENT CHILD]. May I know the reason why not? MULTIPLE CODING POSSIBLE

आपने बताया कि आपने कभी ................. को लिए पैकेज्ड शिशु अनाज/ शिशु आहार को सर्व नही किया। क्या मै कारण जान सकता हूँ?

\begin{tabular}{|c|c|c|}
\hline & & Q319 \\
\hline I cannot afford packaged infant cereal/ baby food & $\begin{array}{l}\text { मै पैकेज्ड शिशु अनाज/ शिशु आहार का } \\
\text { वहन/ अफोर्ड नही कर सकता है }\end{array}$ & 01 \\
\hline It is too expensive as compared to homemade food & $\begin{array}{r}\text { यह घर पर बने आहार की तुलना मे बहुत } \\
\text { महंगा है }\end{array}$ & 02 \\
\hline $\begin{array}{r}\text { There is no small pack to try it and if my child does not eat } \\
\text { it, it will be a waste of money }\end{array}$ & $\begin{array}{l}\text { इसे आजमाने के लिए छोटा पैक नही है और } \\
\text { यदि मेरा बच्चा इसे नही खाता, यह पैसे की } \\
\text { बर्बादी होगी }\end{array}$ & 03 \\
\hline It's not healthy for my child & यह मेरे बच्चे के लिए स्वार्थ्यदायक नही है & 04 \\
\hline My child doesn't like it & मेरा बच्चा इसे पसंद नहीं करता है & 06 \\
\hline My child is too young for it & मेरे बच्चा इसके लिए बहुत छोटा है & 07 \\
\hline My child is too old for it & मेरा बच्चा इसके लिए बहुत बडा है & 08 \\
\hline My child gets digestive problem after eating it & $\begin{array}{l}\text { इसे खाने के बाद मेरे बच्चे को पाचक } \\
\text { परेशानी हो गयी }\end{array}$ & 09 \\
\hline $\begin{array}{r}\text { Homemade food has much more variety and child requires } \\
\text { variety }\end{array}$ & $\begin{array}{r}\text { घर पर बने आहार मे ज्यादा वैरायटी होती है } \\
\text { और बच्चे को वैरायटी चाहिए }\end{array}$ & 12 \\
\hline It does not provide fat & यह फैट प्रदान नही करता है & 13 \\
\hline $\begin{array}{r}\text { My mother-in-law/ other family members/ friends are } \\
\text { against it }\end{array}$ & $\begin{array}{r}\text { मेरी सास/अन्य परिवार के सदस्य/दोस्त इसके } \\
\text { विरोध में हैं }\end{array}$ & 26 \\
\hline $\begin{array}{r}\text { The doctor advised me not to feed packaged food to my } \\
\text { child }\end{array}$ & $\begin{array}{l}\text { डॉक्टर ने मेरे बच्चे को पैकेज्ड आहार नही } \\
\text { खिलाने की सलाह दी थी }\end{array}$ & 16 \\
\hline Do not have knowledge about all benefits it provides & $\begin{array}{l}\text { इसके द्वारा दिये जाने वाले सभी फायदों के } \\
\text { बारे में नहीं जानती }\end{array}$ & 17 \\
\hline $\begin{array}{r}\text { It cannot be prepared and kept for a few hours like home } \\
\text { food, it has to be eaten immediately }\end{array}$ & $\begin{array}{l}\text { इसे घर पर बने आहार की तरह बनाकर कुछ } \\
\text { घंटो के लिए नही रखा जा सकता, इसे तुरंत } \\
\text { खाना होता है }\end{array}$ & 18 \\
\hline It is raw/ it is not made by boiling or cooking the food & $\begin{array}{l}\text { यह कच्चा है/ यह खाना को पकाने या } \\
\text { उबालने द्वारा नही बनाया जाता }\end{array}$ & 19 \\
\hline It may contain preservatives which are harmful & $\begin{array}{l}\text { इसमे प्रतिरक्षक/प्रिजर्वेटिव हो सकते है जो } \\
\text { हानिकारक है }\end{array}$ & 20 \\
\hline $\begin{array}{r}\text { The child will not develop habit of eating homemade food } \\
\text { for later in life }\end{array}$ & $\begin{array}{l}\text { जीवन मे बाद के लिए बच्चे मे घर पर बने } \\
\text { आहार को खाने की आदत विकसित नही } \\
\text { होगी }\end{array}$ & 22 \\
\hline It is very sticky and sticks into the mouth of the child & $\begin{array}{l}\text { यह बहुत चिपचिपा है और बच्चे के मुहं मे } \\
\text { चिपकता है }\end{array}$ & 24 \\
\hline I feel guilty when I give packaged food to my child & $\begin{array}{l}\text { मेरे बच्चे को पैकेज्ड आहार देते समय मै } \\
\text { दोषी महसूस करती हूँ }\end{array}$ & 25 \\
\hline Not available in the places where I go for shopping & $\begin{array}{l}\text { उस जगह पर उपलब्ध नही जहां से मै } \\
\text { खरीदारी करने के लिए जाती हूँ }\end{array}$ & 05 \\
\hline Any other (please specify) & कोई अन्य (कृप्या स्पष्ट करे) & \\
\hline Any other (please specify) & कोई अन्य (कृप्या स्पष्ट करे) & \\
\hline
\end{tabular}

Q320 Would you buy packaged infant cereals/ baby foods if you could afford it? SINGLE CODING ONLY

क्या आप पैकेज्ड शिशु अनाज/ शिशु आहार को खरीदेंगें यदि आप इसका वहन/अफोर्ड कर सके ?

\begin{tabular}{|c|c|c|}
\hline Yes हाँ & 01 & \multirow{3}{*}{$\Rightarrow$ GO TO Q324 } \\
\hline No नही & 02 & \\
\hline $\begin{array}{r}\text { Don't know (DO NOT READ) पता } \\
\text { नही }\end{array}$ & 98 & \\
\hline
\end{tabular}

\section{ASK Q321 IF '1' CODED IN Q318}

Q321 Have you ever bought packaged infant cereals/ baby foods for [REFERENT CHILD]? SINGLE CODING ONLY Assign 10 class-points if YES (circle number): क्या आपने कभी ............... के लिए पैकेज्ड शिशु अनाज/ शिशु आहार को खरीदा है ?

\begin{tabular}{|r|c|c|}
\hline Yes हाँ & 01 & $\rightarrow$ GO TO Q322 \\
\hline No नही & 02 & $\rightarrow$ GO TO Q323 \\
\hline Don't know (DO NOT READ) पता & 98 & $\rightarrow$ GO TO Q322 \\
\hline
\end{tabular}


ASK Q322 IF PACKAGED INFANT CEREAL (155) NOT CODED IN Q301 AND ‘1'l'98' CODED IN Q321

Q322 SHOWCARD Q322 Could you please tell me why you do not give packaged infant cereals/ baby foods to [REFERENT CHILD] more often? MULTIPLE CODING POSSIBLE

कृप्या मुझे बताए आप .............. के लिए ज्यादातर पैकेज्ड शिशु अनाज/ शिशु खाद्य क्यों नही देते है ?

\begin{tabular}{|c|c|c|}
\hline & & Q322 \\
\hline I cannot afford packaged infant cereal/ baby food & $\begin{array}{l}\text { मै पैकेज्ड शिशु अनाज/ शिशु आहार का वहन/ } \\
\text { अफोर्ड नही कर सकता है }\end{array}$ & 01 \\
\hline It is too expensive as compared to homemade food & $\begin{array}{r}\text { यह घर पर बने आहार की तुलना मे बहुत } \\
\text { महंगा है }\end{array}$ & 02 \\
\hline $\begin{array}{r}\text { There is no small pack to try it and if my child does } \\
\text { not eat it, it will be a waste of money }\end{array}$ & $\begin{array}{l}\text { इसे आजमाने के लिए छोटा पैक नही है और } \\
\text { यदि मेरा बच्चा इसे नही खाता, यह पैसे की } \\
\text { बर्बादी होगी }\end{array}$ & 03 \\
\hline It's not healthy for my child & यह मेरे बच्चे के लिए स्वास्थ्यदायक नही है & 04 \\
\hline My child doesn't like it & मेरा बच्चा इसे पसंद नहीं करता है & 06 \\
\hline My child is too young for it & मेरे बच्चा इसके लिए बहुत छोटा है & 07 \\
\hline My child is too old for it & मेरा बच्चा इसके लिए बहुत बडा है & 08 \\
\hline My child gets digestive problem after eating it & $\begin{array}{r}\text { इसे खाने के बाद मेरे बच्चे को पाचक परेशानी } \\
\text { हो गयी }\end{array}$ & 09 \\
\hline $\begin{array}{r}\text { Homemade food has much more variety and child } \\
\text { requires variety }\end{array}$ & $\begin{array}{r}\text { घर पर बने आहार मे ज्यादा वैरायटी होती है } \\
\text { और बच्चे को वैरायटी चाहिए }\end{array}$ & 12 \\
\hline It does not provide fat & यह फैट प्रदान नही करता है & 13 \\
\hline $\begin{array}{r}\text { My mother-in-law/ other family members/ friends are } \\
\text { against it }\end{array}$ & $\begin{array}{r}\text { मेरी सास/अन्य परिवार के सदस्य/दोस्त इसके } \\
\text { विरोध में हैं }\end{array}$ & 26 \\
\hline $\begin{array}{r}\text { The doctor advised me not to feed packaged food to } \\
\text { my child }\end{array}$ & $\begin{array}{l}\text { डॉक्टर ने मेरे बच्चे को पैकेज्ड आहार नही } \\
\text { खिलाने की सलाह दी थी }\end{array}$ & 16 \\
\hline Do not have knowledge about all benefits it provides & $\begin{array}{r}\text { इसके द्वारा दिये जाने वाले सभी फायदों के बारे } \\
\text { में नहीं जानती }\end{array}$ & 17 \\
\hline $\begin{array}{l}\text { It cannot be prepared and kept for a few hours like } \\
\text { home food, it has to be eaten immediately }\end{array}$ & $\begin{array}{l}\text { इसे घर पर बने आहार की तरह बनाकर कुछ } \\
\text { घंटो के लिए नही रखा जा सकता, इसे तुरंत } \\
\text { खाना होता है }\end{array}$ & 18 \\
\hline It is raw/ it is not made by boiling or cooking the food & $\begin{array}{l}\text { यह कच्चा है/ यह खाना को पकाने या उबालने } \\
\text { द्वारा नही बनाया जाता }\end{array}$ & 19 \\
\hline It may contain preservatives which are harmful & $\begin{array}{l}\text { इसमे प्रतिरक्षक/प्रिजर्वेटिव हो सकते है जो } \\
\text { हानिकारक है }\end{array}$ & 20 \\
\hline $\begin{array}{r}\text { The child will not develop habit of eating homemade } \\
\text { food for later in life }\end{array}$ & $\begin{array}{l}\text { जीवन मे बाद के लिए बच्चे मे घर पर बने } \\
\text { आहार को खाने की आदत विकसित नही होगी }\end{array}$ & 22 \\
\hline It is very sticky and sticks into the mouth of the child & $\begin{array}{r}\text { यह बहुत चिपचिपा है और बच्चे के मुहं मे } \\
\text { चिपकता है }\end{array}$ & 24 \\
\hline I feel guilty when I give packaged food to my child & $\begin{array}{l}\text { मेरे बच्चे को पैकेज्ड आहार देते समय मै दोषी } \\
\text { महसूस करती हूँ }\end{array}$ & 25 \\
\hline Not available in the places where I go for shopping & $\begin{array}{l}\text { उस जगह पर उपलब्ध नही जहां से मै खरीदारी } \\
\text { करने के लिए जाती हूँ }\end{array}$ & 05 \\
\hline Any other (please specify) & कोई अन्य (कृप्या स्पष्ट करे) & \\
\hline Any other (please specify) & कोई अन्य (कृप्या स्पष्ट करे) & \\
\hline
\end{tabular}


ASK Q323 IF PACKAGED INFANT CEREAL (155) NOT CODED IN Q301 AND '2' CODED IN Q321

Q323 SHOWCARD Q323 You said that you never bought packaged infant cereals/ baby foods for [REFERENT CHILD]. May I know the reason why not? MULTIPLE CODING POSSIBLE

आपने कहा कि आपने ........... के लिए कभी भी पैकेज्ड शिशु अनाज/ शिशु आहार को नही खरीदाहै । क्या मै कारण जान सकता हूँ क्यों नही ?

\begin{tabular}{|c|c|c|}
\hline & & Q323 \\
\hline $\begin{array}{r}\text { I cannot afford packaged infant } \\
\text { cereal/ baby food }\end{array}$ & $\begin{array}{r}\text { मै पैकेज्ड शिशु अनाज/ शिशु आहार का वहन/ अफोर्ड } \\
\text { नही कर सकता है }\end{array}$ & 01 \\
\hline $\begin{array}{r}\text { It is too expensive as compared to } \\
\text { homemade food }\end{array}$ & यह घर पर बने आहार की तुलना मे बहुत महंगा है & 02 \\
\hline $\begin{array}{r}\text { There is no small pack to try it and if } \\
\text { my child does not eat it, it will be a } \\
\text { waste of money }\end{array}$ & $\begin{array}{l}\text { इसे आजमाने के लिए छोटा पैक नही है और यदि मेरा } \\
\text { बच्चा इसे नही खाता, यह पैसे की बर्बादी होगी }\end{array}$ & 03 \\
\hline It's not healthy for my child & यह मेरे बच्चे के लिए स्वास्थ्यदायक नही है & 04 \\
\hline My child doesn't like it & मेरा बच्चा इसे पसंद नहीं करता है & 06 \\
\hline My child is too young for it & मेरे बच्चा इसके लिए बहुत छोटा है & 07 \\
\hline My child is too old for it & मेरा बच्चा इसके लिए बहुत बडा है & 08 \\
\hline $\begin{array}{r}\text { My child gets digestive problem after } \\
\text { eating it }\end{array}$ & इसे खाने के बाद मेरे बच्चे को पाचक परेशानी हो गयी & 09 \\
\hline $\begin{array}{l}\text { Homemade food has much more } \\
\text { variety and child requires variety }\end{array}$ & $\begin{array}{r}\text { घर पर बने आहार मे ज्यादा वैरायटी होती है और बच्चे } \\
\text { को वैरायटी चाहिए }\end{array}$ & 12 \\
\hline It does not provide fat & यह फैट प्रदान नही करता है & 13 \\
\hline $\begin{array}{l}\text { My mother-in-law/ other family } \\
\text { members/ friends are against it }\end{array}$ & $\begin{array}{l}\text { मेरी सास/अन्य परिवार के सदस्य/दोस्त इसके विरोध में } \\
\text { हैं }\end{array}$ & 26 \\
\hline $\begin{array}{l}\text { The doctor advised me not to feed } \\
\text { packaged food to my child }\end{array}$ & $\begin{array}{l}\text { डॉक्टर ने मेरे बच्चे को पैकेज्ड आहार नही खिलाने की } \\
\text { सलाह दी थी }\end{array}$ & 16 \\
\hline $\begin{array}{r}\text { Do not have knowledge about all } \\
\text { benefits it provides }\end{array}$ & $\begin{array}{l}\text { इसके द्वारा दिये जाने वाले सभी फायदों के बारे में नहीं } \\
\text { जानती }\end{array}$ & 17 \\
\hline $\begin{array}{r}\text { It cannot be prepared and kept for a } \\
\text { few hours like home food, it has to be } \\
\text { eaten immediately }\end{array}$ & $\begin{array}{l}\text { इसे घर पर बने आहार की तरह बनाकर कुछ घंटो के } \\
\text { लिए नही रखा जा सकता, इसे तुरंत खाना होता है }\end{array}$ & 18 \\
\hline $\begin{array}{r}\text { It is raw/ it is not made by boiling or } \\
\text { cooking the food }\end{array}$ & $\begin{array}{r}\text { यह कच्चा है/ यह खाना को पकाने या उबालने द्वारा नही } \\
\text { बनाया जाता }\end{array}$ & 19 \\
\hline $\begin{array}{r}\text { It may contain preservatives which } \\
\text { are harmful }\end{array}$ & इसमे प्रतिरक्षक/प्रिजर्वेटिव हो सकते है जो हानिकारक है & 20 \\
\hline $\begin{array}{l}\text { The child will not develop habit of } \\
\text { eating homemade food for later in life }\end{array}$ & $\begin{array}{l}\text { जीवन मे बाद के लिए बच्चे मे घर पर बने आहार को } \\
\text { खाने की आदत विकसित नही होगी }\end{array}$ & 22 \\
\hline $\begin{array}{r}\text { It is very sticky and sticks into the } \\
\text { mouth of the child }\end{array}$ & यह बहुत चिपचिपा है और बच्चे के मुहं मे चिपकता है & 24 \\
\hline $\begin{array}{r}\text { I feel guilty when I give packaged food } \\
\text { to my child }\end{array}$ & $\begin{array}{l}\text { मेरे बच्चे को पैकेज्ड आहार देते समय मै दोषी महसूस } \\
\text { करती हूँ }\end{array}$ & 25 \\
\hline $\begin{array}{r}\text { Not available in the places where I go } \\
\text { for shopping }\end{array}$ & $\begin{array}{r}\text { उस जगह पर उपलब्ध नही जहां से मै खरीदारी करने के } \\
\text { लिए जाती हूँ }\end{array}$ & 05 \\
\hline Any other (please specify) & कोई अन्य (कृप्या स्पष्ट करे) & \\
\hline Any other (please specify) & कोई अन्य (कृप्या स्पष्ट करे) & \\
\hline
\end{tabular}

ASK ALL

Q324 Please tell me does your household receive packaged infant cereals/ baby foods from social programs? SINGLE CODING ONLY

कृप्या मुझे बताए क्या आपका परिवार किसी समाजिक प्रोग्राम से पैकेज्ड शिशु अनाज/ शिशु आहार को प्राप्त करता है ?

There are various food related social programs run by government or any social organization. Under these programs, food is provided to a household directly or indirectly generally either at no cost or at subsidized cost. Some examples of such programs are ration programs, school feeding programs, cash transfers for food etc.

सरकार या अन्य समाजिक संगठनो द्वारा विभिन्न खाद्य संबंधित समाजिक कार्यकम चलाए जा रहे है। इन प्रोग्राम के अंतर्गत खाना सीधे एक परिवार को या बिना किसी लागत या कम की गयी कीमत पर परिवारो को दिया जाता है। ऐसे प्रोग्रामो के कुछ कुछ उदाहरण है राशन प्रोग्राम, स्कूल आहार प्रोग्राम, आहार के लिए नगद हस्तांतरण इत्यादि।

\begin{tabular}{|c|c|c|}
\hline Yes हाँ & 1 & $\rightarrow$ GO TO Q325 \\
\hline No नही & 2 & $\rightarrow$ GO TO Q400 \\
\hline \multicolumn{3}{|c}{1363}
\end{tabular}

\section{ASK Q325 IF ' 1 'l'YES' CODED IN Q324}

Q325 From which program did you receive the packaged infant cereals/ baby foods you gave to [REFERENT CHILD]? RECORD VERBATIM 
किस प्रोग्राम से आपने को देने के लिए पैकेज्ड शिशु अनाज/ शिशु आहार को प्राप्त किया था ?

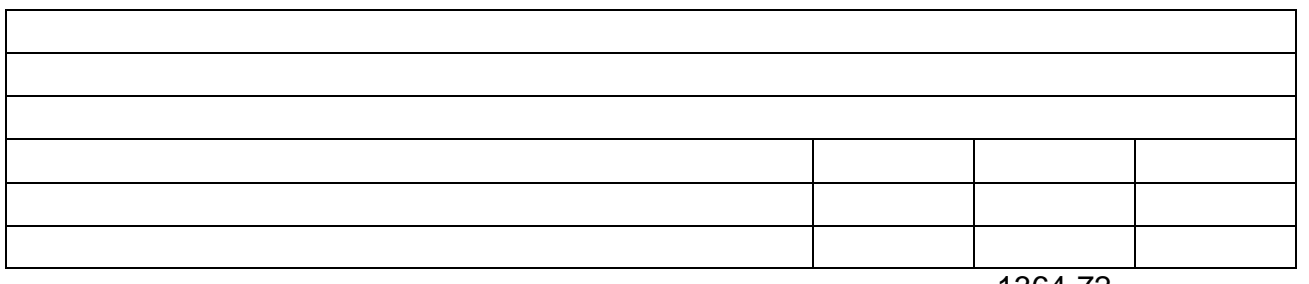

1364-72

\section{PRICE SENSITIVITY SECTION}

\section{TO NON BUYERS ASK ONLY APPROACH 2}

\section{FOR CURRENT BUYERS, SELECT FROM RANDOM LIST WHETHER APPROACH 1 OR APPROACH 2 COMES FIRST}

\section{GRID ONLY FOR CURRENT BUYERS}

PRICING APPROACH PANELS (SINGLE CODING ONLY) See number on RANDOM LIST.

\begin{tabular}{|c|c|c|c|c|}
\hline Panel 1 & ASKED FIRST & Approach 1 \\
(start at Q401) & $\begin{array}{c}\text { Approach 2 } \\
\text { (start at Q420) }\end{array}$ & 1 & \multirow{2}{*}{1477} \\
Panel 2 & $\begin{array}{c}\text { Approach 2 } \\
\text { (start at Q420) }\end{array}$ & $\begin{array}{c}\text { Approach 1 } \\
\text { (start at Q401) }\end{array}$ & 2 & \\
\hline
\end{tabular}

\section{APPROACH 1}

\section{ASK 0401-0415 TO CURRENT BUYERS}

\section{DETERMINE CURRENT BRAND}

Brand (Q307a):

Pack Type (Pack type of brand coded in Q307a):

Pack Size (Pack size of brand coded in Q307a):

Q401 You mentioned that you serve [BRAND, PACK TYPE AND PACK SIZE] most often to your child. Please tell me at what price do buy each pack of this brand, pack type and size? RECORD VERBATIM WITH LEADING ZEROES आपने बताया कि आपके बच्चे के लिए आप ज्यादातर ......... सर्व करती है। कृप्या मुझे बताए आप इस ब्रांड, पैक प्रकार और साइज के प्रत्येक पैक को किस कीमत पर खरीदती है ?

\begin{tabular}{|r|l|l|l|l|}
\hline PRICE OF MOST OFTEN & & & & \\
USED PACK (in Rs.) & & & & \\
\hline
\end{tabular}

Q402 How many packs of [BRAND, PACK TYPE AND PACK SIZE] do you buy in a usual shopping trip? RECORD VERBATIM WITH LEADING ZEORES

के कितने पैक्स को आप सामान्य खरीदारी मे खरीदती है ?

\begin{tabular}{|r|l|l|}
\hline NUMBER OF PACKS PER & $1482-83$ \\
SHOPPING TRIP & & \\
\hline
\end{tabular}

Q403 How often do you make such shopping trips? RECORD VERBATIM WITH LEADING ZEROES FOR NUMBER OF DAYS BETWEEN SHOPPING TRIPS

आप ऐसी खरीदारी कितनी बार करती है ?

\begin{tabular}{|r|l|l|}
\hline NUMBER OF DAYS BETWEEN & & \\
SHOPPING TRIPS & & \\
\hline
\end{tabular}




\begin{tabular}{|c|c|}
\hline $20 \%$ & 1 \\
\hline $40 \%$ & 2 \\
\hline $60 \%$ & 3 \\
\hline $80 \%$ & 4 \\
\hline
\end{tabular}

\section{Q405 COMPUTE DISCOUNTED PRICE:}

\begin{tabular}{|c|c|c|c|c|c|}
\hline DISCOUNT SELECTED & 1 & 2 & 3 & 4 & \\
\hline Brand & DISCOUNT - $20 \%$ & DISCOUNT - $40 \%$ & DISCOUNT - $60 \%$ & DISCOUNT - $80 \%$ & $\begin{array}{l}\text { DISCOUNTED } \\
\text { PRICE }\end{array}$ \\
\hline $\begin{array}{l}\text { Price Paid per Pack } \\
\text { (Q401) }\end{array}$ & $\longleftarrow \times 0.8$ & $\ldots 0.6$ & $\ldots \times 0.4$ & $\ldots \times 0.2$ & \\
\hline
\end{tabular}

1511-16

\begin{tabular}{|c|c|c|c|c|c|}
\hline $\begin{array}{l}\text { Brand - Cerelac - } 1000 \mathrm{~g} \\
\text { Price paid per pack - Rs. } \\
\text { Discount Selected - '2'। }\end{array}$ & $\begin{array}{l}m \\
\text { count } 40 \%\end{array}$ & & & & \\
\hline DISCOUNTED PRICE & $\begin{array}{l}365 \times 0.6 \\
\text { Rs. } 219\end{array}$ & & & & \\
\hline DISCOUNT SELECTED & 1 & (2) & 3 & 4 & \\
\hline Brand Cerelac $1000 \mathrm{gm}$ & DISCOUNT - 20\% & DISCOUNT - 40\% & DISCOUNT - 60\% & DISCOUNT - 80\% & $\begin{array}{l}\text { DISCOUNTED } \\
\text { PRICE }\end{array}$ \\
\hline $\begin{array}{l}\text { Price Paid per Pack } \\
\text { (Q401) Rs. } 365\end{array}$ & $\ldots 0.8$ & $\ldots 0.6$ & $\ldots 0.4$ & $\ldots 0.2$ & Rs. 219 \\
\hline
\end{tabular}

\section{ASK Q406-415 FOR DISCOUNTED PRICE}

SAY: We are interested in finding out how much packaged infant cereal/ baby food you would buy at a lower price. This lower price would be a permanently lower price and not just a temporary promotion price. At this price, product is going to be regularly available in the market हम यह पता लगाने मे दिलचस्प है कि आप कम कीमत पर कितना पैकेज्ड शिशु अनाज/ शिशु आहार खरीदेंगी। कम कीमत स्थायी कम कीमत होगी और एक अस्थायी प्रोमोशन कीमत नही। इस कीमत पर, प्रोडक्ट बाजार मे नियमित रूप से उपलब्ध होगा।

Q406 SHOWCARD 406 please give me your opinion on value for money of this packaged infant cereal/ baby food if one package of this was available at [DISCOUNTED PRICE]? SINGLE CODING ONLY

कृष्या मुझे इस पैकेज्ड शिशु अनाज/ शिशु आहार की पैसे के लिए वसुली पर अपनी राय दे यदि इसका एक पैकेज

\begin{tabular}{|r|r|c|}
\hline Excellent & सर्वोत्तम & 05 \\
\hline Very good & बहुत अच्छा & 04 \\
\hline Good & अच्छा & 03 \\
\hline Fair & ठीक & 02 \\
\hline Poor & खराब & 01 \\
\hline Don't know (DO NOT SHOW) & पता नही & 98 \\
\hline
\end{tabular}

Q407 SHOWCARD 407 please give tell me how willing are you to buy this packaged infant cereal/ baby food in future if one package of this was available at [DISCOUNTED PRICE]? SINGLE CODING ONLY

कृप्या मुझे बताए आपकी भविष्य मे इस पैकेज्ड शिशु अनाज/ शिशु आहार को खरीदने की कितनी संभावना है यदि इसका एक पैकेज ............ पर उपलब्ध हो ?

\begin{tabular}{|c|c|c|c|}
\hline Will definitely buy & निश्चित रूप से खरीदेंगें & 05 & \multirow{4}{*}{$\rightarrow$ GO TO 408} \\
\hline Will probably buy & शायद खरीदेंगें & 04 & \\
\hline May or may not buy & $\begin{array}{r}\text { खरीद भी सकते है या } \\
\text { नही भी }\end{array}$ & 03 & \\
\hline Will probably not buy & शायद नही खरीदेंगें & 02 & \\
\hline Will definitely not buy & $\begin{array}{l}\text { निश्चित रूप से नही } \\
\text { खरीदेंगें }\end{array}$ & 01 & $\rightarrow$ GO TO 501 OR APP 2 \\
\hline Don't know (DO NOT SHOW) & पता नही & 98 & $\rightarrow$ GO TO 408 \\
\hline
\end{tabular}

\section{ASK Q408 IF CODED '2'l'3'/'4'/'5'/'98' CODED IN Q407}

Q408 How many packs of [BRAND NAME, PACK TYPE AND PACK SIZE] would you buy in a usual shopping trip if the price of a package was [DISCOUNTED PRICE]? RECORD VERBATIM WITH LEADING ZEROES
के

हो ? 
How often would you make such shopping trips? RECORD VERBATIM WITH LEADING ZEROES FOR NUMBER OF DAYS BETWEEN SHOPPING TRIPS

आप ऐसी खरीदारी कितनी बार करेंगी ?

$$
\begin{array}{r}
\text { NUMBER OF DAYS BETWEEN } \\
\text { SHOPPING TRIPS }
\end{array}
$$

Q410 Will you serve more packaged infant cereal/ baby food as a result of this lower price or will consumption remain the same? SINGLE CODING ONLY

क्या आप कम कीमत के परिणाम स्वरूप ज्यादा पैकेज्ड शिशु अनाज/ शिशु आहार को सर्व करेंगी या खपत समान रहेगी ?

\begin{tabular}{|c|c|c|c|}
\hline $\begin{array}{r}\text { Yes, will serve more packaged } \\
\text { infant cereal }\end{array}$ & $\begin{array}{l}\text { हाँ, ज्यादा पैकेज्ड शिशु अनाज } \\
\text { सर्व करेंगें }\end{array}$ & 01 & $\rightarrow$ GO TO 411 \\
\hline $\begin{array}{l}\text { No, will NOT serve more packaged infant } \\
\text { cereal/ Consumption will remain the same }\end{array}$ & $\begin{array}{l}\text { नही, पैकेज्ड शिशु अनाज ज्यादा } \\
\text { सर्व नही करेंगें / खपत समान } \\
\text { रहेगी }\end{array}$ & 02 & $\rightarrow$ GO TO 501 OR APP 2 \\
\hline Don't know (DO NOT SHOW) & पता नही & 98 & $\rightarrow$ GO TO 411 \\
\hline
\end{tabular}

\section{ASK Q411 IF CODED ‘1' IN Q410}

Q411 SHOWCARD Q411 If you will serve more packaged infant cereal/ baby food that you currently do, who would consume the ADDITIONAL packaged infant cereal/ baby food? SINGLE CODING ONLY

यदि आप वर्तमान से ज्यादा पैकेज्ड शिशु अनाज/ शिशु आहार को सर्व करेंगी, तो कौन अतिरिक्त पैकेज्ड शिशु अनाज/ शिशु आहार को खाएगा ?

\begin{tabular}{|c|c|c|c|}
\hline $\begin{array}{r}\text { Equally distributed among adults and } \\
\text { children }\end{array}$ & $\begin{array}{r}\text { व्यस्को और बच्चो के बीच बराबर } \\
\text { बांटा था }\end{array}$ & 01 & $\rightarrow$ GO TO 412 \\
\hline Only adults & केवल व्यस्क & 02 & \multirow{2}{*}{$\rightarrow$ GO TO 501 OR APP 2} \\
\hline Mainly adults & मुख्य रूप से व्यस्क & 03 & \\
\hline Mainly children & मुख्य रूप से बच्चे & 04 & \multirow{2}{*}{$\rightarrow$ GO TO 412} \\
\hline Only child/ children & केवल बच्चा/ बच्चे & 05 & \\
\hline Don't know (DO NOT SHOW) & पता नही & 98 & $\rightarrow$ GO TO 501 OR APP 2 \\
\hline
\end{tabular}

ASK Q412 IF '1'l'4'l'5' CODED IN Q411

Q412 SHOWCARD Q412 How will the ADDITIONAL packaged infant cereal/ baby food be distributed among the children? SINGLE CODING ONLY

अतिरिक्त पैकेज्ड शिशु अनाज/ शिशु आहार को बच्चो के बीच कैसे बट्वारा किया जाएगा ?

IF ONLY 1 CHILD IN THE FAMILY, AUTOCODE ' 2 '

\begin{tabular}{|c|c|c|c|}
\hline Equally distributed among all children & $\begin{array}{r}\text { सभी बच्चो के बीच बराबर } \\
\text { बांटा था }\end{array}$ & 01 & \multirow{4}{*}{$\rightarrow$ GO TO 413} \\
\hline Only [REFERENT CHILD] & केवल $\quad . . . . . . . . . . .$. & 02 & \\
\hline Mainly [REFERENT CHILD] & मुख्य रूप से .......... & 03 & \\
\hline Mainly the other children & मुख्य रूप से अन्य बच्चे & 04 & \\
\hline Only the other children & केवल अन्य बच्चे & 05 & $\rightarrow$ GO TO 501 OR APP 2 \\
\hline Don't know (DO NOT SHOW) & पता नही & 98 & $\rightarrow$ GO TO 413 \\
\hline
\end{tabular}

ASK Q413 IF '1'l'2'l'3'l'4'l'98' CODED IN Q412

Q413 Would you feed less of other food and drinks for [REFERENT CHILD]? SINGLE CODING ONLY क्या आप ........... को अन्य आहार और ड्रिंक्स कम खिलायेंगें/ पिलायेंगें ?

\begin{tabular}{|l|c|l|}
\hline Yes हाँ & 1 & $\rightarrow$ GO TO 414 \\
\hline No नही & 2 & \multirow{2}{*}{$\rightarrow$ GO TO 501 OR APP 2} \\
$\begin{array}{l}\text { Don't know (DO NOT SHOW) पता } \\
\text { नही }\end{array}$ & 98 & \\
\hline
\end{tabular}

ASK Q414 IF '1' / 'YES' CODED IN Q413

Q414 SHOWCARD Q414 What kind of food and drinks would you feed less to [REFERENT CHILD]? MULTIPLE CODING POSSIBLE

आप ............ के लिए किस प्रकार के आहार और ड्रिंक्स को कम खिलायेंगें। पिलायेंगें ?

\begin{tabular}{|l|l|l|}
\hline Breast Milk & माँ का दूध 01 \\
\hline Cereals like Daliya/ Khichdi/ Mixed Grains/ Sewiya & $\begin{array}{l}\text { सीरियल्स जैसे दलिया/खिचड़ी/मिले } \\
\text { अनाज/सेवईंया }\end{array}$ \\
\hline Cereals like Ragi/ Barleyl Bajra/ Maize/ Corn & सिरिल्स जैसे रागी/जों/बाजरा/मक्का/दाना & 03 \\
\hline Oats/ Corn flakes/ Rice flakes & ओट्स/ कोर्नफ्लेक्स/ चावल के फ्लेक्स \\
\hline
\end{tabular}




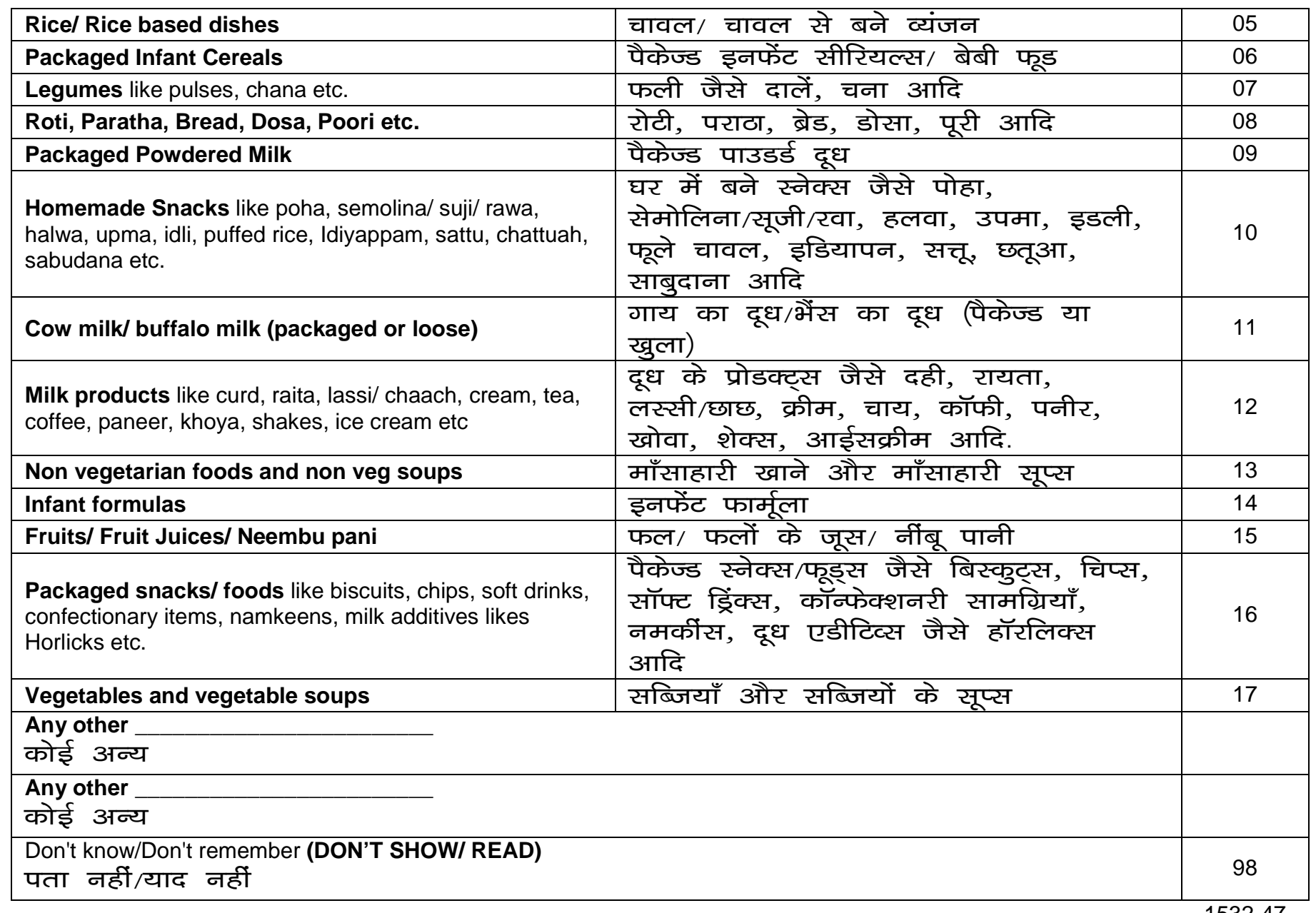

Q415 Please tell me whether you would buy less of other brands of packaged infant cereals/ baby food that you buy regularly? MULTIPLE CODING POSSIBLE

कृप्या मुझे बताए क्या आप पैकेज्ड शिशु अनाज/ शिशु आहार के अन्य ब्रांडस को कम खरीदेंगों जो कि आप नियमित रूप से खरीदते है ?

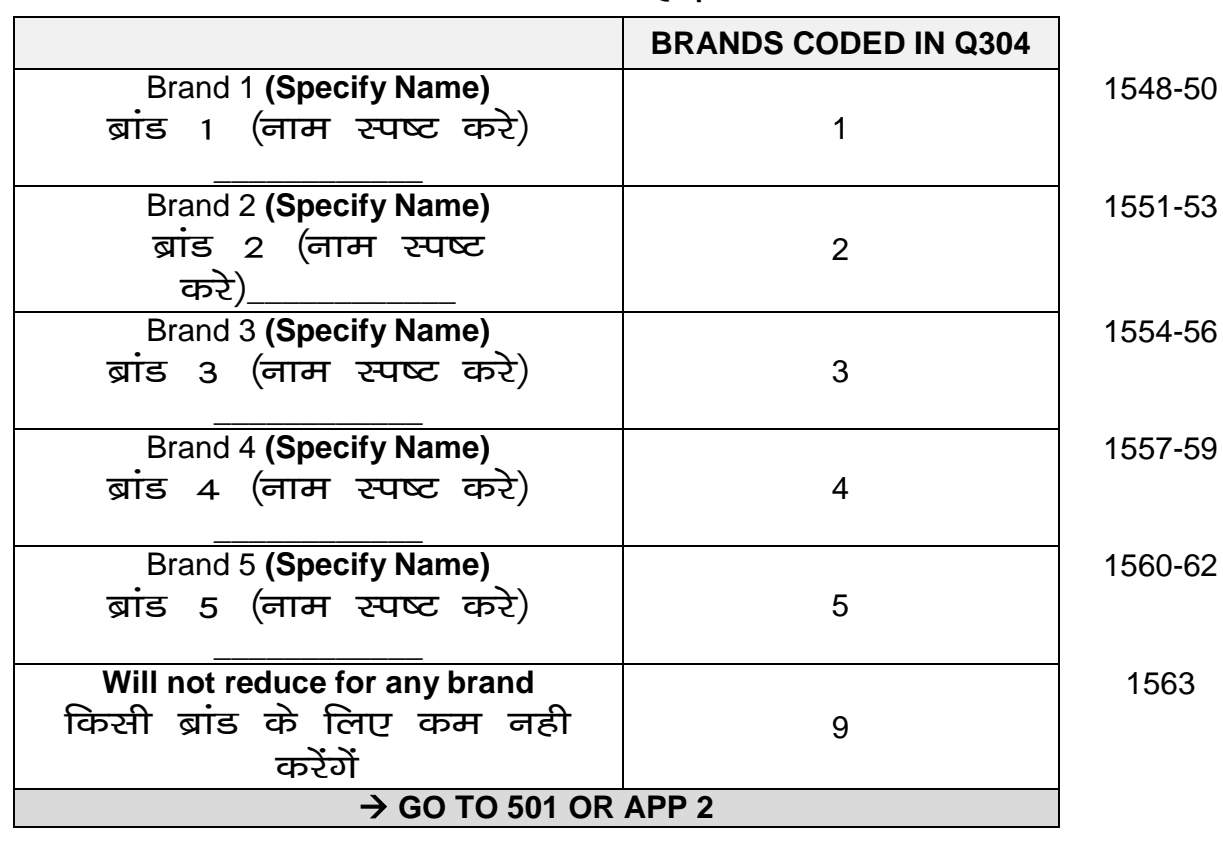




\section{APPROACH 2- ASK ALL}

SAY: Suppose you were eligible for a program allowing you, as the mother of a child aged 6-23 months old, to buy packaged infant cereal/ baby food at a reduced price. Each pack of this special infant cereal for children 6-23 months of age would be sufficient to prepare 1 serving of cereals for [REFERENT CHILD]. The required daily amount of this infant cereal is 2 servings per day i.e. 2 packs per day.

कहेः कल्पना करे आप एक प्रोग्राम के लिए योग्य है जो आपको 6-23 महीनो की उम्र के बच्चे की माँ के रूप मे, पैकेज्ड शिशु अनाज/ शिशु आहार एक कम की गयी कीमत पर खरीदने की अनुमति देता है। इस विशेष शिशु अनाज का प्रत्येक पैक $6-23$ महीनो की उम्र के बच्चे के लिए प्रति दिन अनाज की 1 सर्विग बनाने के लिए पर्याप्त होगा। इस शिशु अनाज की प्रति दिन की ज़रूरी माआ प्रति दिन 2 सर्विग है मतलब प्रति दिन 2 पैक।

Please note that this product is not a substitute for breastfeeding and that [REFERENT CHILD] will still require other food items along with this.

कृपया ध्यान रहे कि यह प्रोडक्ट स्तनपान का विकल्प नहीं है और ......... को इसके साथ अन्य खाने की सामग्रियों की जरूरत होगी।

This special price would be a permanently lower price and not just a temporary promotion price. At this price, the product is going to be regularly available in the market.

यह विशेष कीमत स्थायी रूप से कम कीमत होगी और केवल एक अस्थायी कीमत नही होगी। इस कीमत पर, प्रोडक्ट नियमित रूप से बाजार मे उपलब्ध होगा।

According to the programme (child 6-23 months of age) should be fed with 2 packages per day until she/ he reaches the age of 2 years. Please note that this packaged infant cereal/ baby food is for children 6 months to less than 2 years old only and is not advisable to be served to others not within this age range.

प्रोग्राम के अनुसार 6-23 महीने की उम्र के बच्चे को प्रतिदिन 2 पैकेज खिलाना चाहिए जब तक वह 2 वर्ष की उम्र तक नही पहुंचे। कृप्या याद रखें कि यह पैकेज्ड शिशु अनाज/ शिशु आहार केवल 6 महीनो से 2 वर्ष से कम उम्र के बच्चे के लिए है और इस रेंज के बाहर के अन्य को सर्व करने के लिए नही है।

We are interested in finding out how much of this packaged infant cereal/ baby food you would buy. हम यह पता लगाने मे दिलचस्प है आप इस पैकेज्ड शिशु अनाज/ शिशु आहार को कितना खरीदेंगें ।

\section{EXPOSE CONCEPT CARD AND DEBRANDED PACKS}

Q420 Select START_PRICE _2 from RANDOM LIST. START WITH THAT PRICE

\begin{tabular}{|c|c|c|c|c|}
\hline \multicolumn{5}{|c|}{ SINGLE CODING ONLY } \\
\hline P1 & P2 & P3 & P4 & P5 \\
Rs. 14 & Rs. 11 & Rs. 8 & Rs. 5 & Rs. 2 \\
\hline 1 & 2 & 3 & 4 & 5 \\
\hline
\end{tabular}

Q421 Would you buy this packaged infant cereal/ baby food if the price of 1 package was [MENTION PRICE]? SINGLE CODING ONLY

क्या आप इस शिशु अनाज/ शिशु आहार को खरीदेंगें यदि 1 पैकेज की कीमत हो ।

\begin{tabular}{|c|c|c|c|}
\hline Yes हाँ & & 1 & $\rightarrow$ GO TO 423 \\
\hline No नही & & 2 & \multirow{2}{*}{$\rightarrow$ GO TO 422} \\
\hline Don't know (DO NOT SHOW & पता नही & 98 & \\
\hline
\end{tabular}

Q422 Would you buy this packaged infant cereal/ baby food if the price of 1 package was [NEXT LOWER PRICE]? If "NO" CONTINUE TO NEXT LOWER PRICE UNTIL LOWEST PRICE. SINGLE CODING ONLY

क्या आप इस शिशु अनाज/ शिशु आहार को खरीदेंगें यदि 1 पैकेज की कीमत हो ।

\begin{tabular}{|r|c|c|c|c|c|}
\hline $\begin{array}{r}\text { CIRCLE THE } \\
\text { STARTING PRICE }\end{array}$ & 1 & 2 & 3 & 4 & 5 \\
\hline $\begin{array}{c}\text { = NEXT LOWER PRICE } \\
\text { TO START_PRICE_2 }\end{array}$ & Rs. 14 & Rs. 11 & Rs. 8 & Rs. 5 & Rs. 2 \\
\hline $\begin{array}{c}\text { CIRCLE THE PRICE AT } \\
\text { WHICH RESPONDENT } \\
\text { SAID 'YES' }\end{array}$ & $\mathbf{1}$ & 2 & 3 & 4 & 5 \\
\hline
\end{tabular}




\section{ASK Q423 FOR PRICE FOR WHICH '1'l'YES' CODED IN Q421 OR Q422 = BUY PRICE 2}

\section{WRITE "BUY PRICE 2 INTO BOX:}

1568-69

Q423 Would you feed less of other food and drinks for [REFERENT CHILD]? SINGLE CODING ONLY क्या आप ............. को अन्य आहार और ड्रिंक्स कम खिलायेंगें। पिलायेंगें ?

\begin{tabular}{|l|c|c|}
\hline Yes हाँ & 1 & $\rightarrow$ GO TO 424 \\
\hline No नही & 2 & \multirow{2}{*}{$\rightarrow$ GO TO 425} \\
\hline Don't know (DO NOT SHOW) पता नही & 98 & \\
\hline \multicolumn{2}{|c|}{$1570-71$}
\end{tabular}

\section{ASK Q424 IF '1' CODED IN Q423}

Q424 SHOWCARD Q424 What kind of food and drinks would you feed less to [REFERENT CHILD]? MULTIPLE CODING POSSIBLE

आप ............. को किस प्रकार का आहार और ड्रिंक्स कम खिलायेंगें। पिलायेंगें ?

\begin{tabular}{|c|c|c|}
\hline Breast Milk & माँ का दूध & 01 \\
\hline $\begin{array}{l}\text { Cereals like Daliya/ Khichdi/ Mixed Grains/ } \\
\text { Sewiya }\end{array}$ & $\begin{array}{l}\text { सीरियल्स जैसे दलिया/खिचड़ी/मिले } \\
\text { अनाज/सेवईंया }\end{array}$ & 02 \\
\hline Cereals like Ragi/ Barleyl Bajra/ Maize/ Corn & सिरियल्स जैसे रागी/जों/बाजरा/मक्का/दाना & 03 \\
\hline Oats/ Corn flakes/ Rice flakes & ओट्स/ कोर्नफ्लेक्स/ चावल के फ्लेक्स & 04 \\
\hline Rice/ Rice based dishes & चावल/ चावल से बने व्यंजन & 05 \\
\hline Packaged Infant Cereals & पैकेज्ड इनफेंट सीरियल्स/ बेबी फूड & 06 \\
\hline Legumes like pulses, chana etc. & फली जैसे दालें, चना आदि & 07 \\
\hline Roti, Paratha, Bread, Dosa, Poori etc. & रोटी, पराठा, ब्रेड, डोसा, पूरी आदि & 08 \\
\hline Packaged Powdered Milk & पैकेज्ड पाउडर्ड दूध & 09 \\
\hline $\begin{array}{l}\text { Homemade Snacks like poha, semolina/ suji/ } \\
\text { rawa, halwa, upma, idli, puffed rice, } \\
\text { Idiyappam, sattu, chattuah, sabudana etc. }\end{array}$ & $\begin{array}{l}\text { घर में बने स्नेक्स जैसे पोहा, } \\
\text { सेमोलिना/सूजी/रवा, हलवा, उपमा, इडली, } \\
\text { फूले चावल, इडियापन, सत्तू, छतूआ, साबुदाना } \\
\text { आदि }\end{array}$ & 10 \\
\hline Cow milk/ buffalo milk (packaged or loose) & गाय का दूध/भेंस का दूध (पैकेज्ड या खुला) & 11 \\
\hline $\begin{array}{l}\text { Milk products like curd, raita, lassi/ chaach, } \\
\text { cream, tea, coffee, paneer, khoya, shakes, ice } \\
\text { cream etc }\end{array}$ & $\begin{array}{l}\text { दूध के प्रोडक्ट्स जैसे दही, रायता, } \\
\text { लस्सी/छाछ, क्रीम, चाय, कॉफी, पनीर, खोवा, } \\
\text { शेक्स, आईसक्रीम आदि. }\end{array}$ & 12 \\
\hline Non vegetarian foods and non veg soups & माँसाहारी खाने और माँसाहारी सूप्स & 13 \\
\hline Infant formulas & इनफेंट फार्मूला & 14 \\
\hline Fruits/ Fruit Juices/ Neembu pani & फल/ फलों के जूस/ नींबू पानी & 15 \\
\hline $\begin{array}{l}\text { Packaged snacks/ foods like biscuits, chips, } \\
\text { soft drinks, confectionary items, namkeens, } \\
\text { milk additives likes Horlicks etc. }\end{array}$ & $\begin{array}{l}\text { पैकेज्ड स्नेक्स/फुड्स जैसे बिस्कुट्स, चिप्स, } \\
\text { सॉफ्ट ड्रिंक्स, कॉन्फेक्शनरी सामग्रियाँ, } \\
\text { नमकीस, दूध एडीटिव्स जेसे हॉरलिक्स आदि }\end{array}$ & 16 \\
\hline Vegetables and vegetable soups & सब्जियाँ और सब्जियों के सूप्स & 17 \\
\hline \multicolumn{3}{|l|}{$\begin{array}{l}\text { Any other } \\
\text { कोई अन्य }\end{array}$} \\
\hline \multicolumn{3}{|l|}{$\begin{array}{l}\text { Any other } \\
\text { कोई अन्य }\end{array}$} \\
\hline \multicolumn{2}{|c|}{$\begin{array}{l}\text { Don't know/Don't remember (DON'T SHOWI READ) } \\
\text { पता नहीं/याद नहीं }\end{array}$} & 98 \\
\hline
\end{tabular}

Q425 SHOWCARD Q425 Who in your household would consume the packaged infant cereal/ baby food? SINGLE CODING ONLY

आपके परिवार मे कौन पैकेज्ड शिशु अनाज/ शिशु आहार को खायेंगा ?

\begin{tabular}{|c|c|c|c|}
\hline Equally distributed among adults and children & $\begin{array}{r}\text { व्यस्को और बच्चो के बीच बराबर } \\
\text { बांटना }\end{array}$ & 01 & $\rightarrow$ GO TO 426 \\
\hline Only adults & केवल व्यस्क & 02 & \multirow{2}{*}{$\rightarrow$ GO TO 427} \\
\hline Mainly adults & मुख्य रूप से व्यस्क & 03 & \\
\hline Mainly children & मुख्य रूप से बच्चे & 04 & \multirow{2}{*}{$\rightarrow$ GO TO 426} \\
\hline Only child/ children & केवल बच्चा/ बच्चे & 05 & \\
\hline Don't know (DO NOT SHOW) & पता नही & 98 & $\rightarrow$ GO TO 427 \\
\hline
\end{tabular}

\section{ASK Q426 IF '1'l'4'l'5' CODED IN Q425}

Q426 SHOWCARD Q426 How will the packaged infant cereal/ baby food be distributed among the children? SINGLE CODING ONLY

बच्चो के बीच पैकेज्ड शिशु अनाज/ शिशु आहार को कैसे बटवारा किया जाएगा ?

IF ONLY 1 CHILD IN THE FAMILY, AUTOCODE '2' 


\begin{tabular}{|r|r|c|}
\hline Only [REFERENT CHILD] & केवल $\ldots \ldots \ldots \ldots$ & 02 \\
\hline Mainly [REFERENT CHILD] & मुख्य रूप से .......... & 03 \\
\hline Mainly the other children & मुख्य रूप से अन्य बच्चे & 04 \\
\hline Only the other children & केवल अन्य बच्चे & 05 \\
\hline Don't know (DO NOT SHOW) & पता नही & 98 \\
\hline
\end{tabular}

ASK ALL

Q427 SHOWCARD Q427 please give me your opinion on value for money of this packaged infant cereal/ baby food if one package of this was available at (PRICE_BUY_2)? SINGLE CODING ONLY कृष्या मुझे इस पैकेज्ड शिशु अनाज/ शिशु आहार की पैसे के लिए वसुली पर अपनी राय दे यदि इसका एक पैकेज पर उपलब्ध था ?

\begin{tabular}{|r|r|c|}
\hline Excellent & सर्वोत्तम & 05 \\
\hline Very good & बहुत अच्छा & 04 \\
\hline Good & अच्छा & 03 \\
\hline Fair & ठीक & 02 \\
\hline Poor & खराब & 01 \\
\hline Don't know (DO NOT SHOW) & पता नही & 98 \\
\hline
\end{tabular}

Q428 SHOWCARD 428 please tell me how willing are you to buy this packaged infant cereal/ baby food in future if one package of this was available at (PRICE_BUY_2)? SINGLE CODING ONLY

कृप्या मुझे बताए आपकी भविष्य मे इस पैकेज्ड शिशु अनाज/ शिशु आहार को खरीदने की कितनी संभावना है यदि इसका एक पैकेज पर उपलब्ध हो ?

\begin{tabular}{|c|c|c|c|}
\hline Will definitely buy & निश्चित रूप से खरीदेंगें & 05 & \multirow{4}{*}{$\rightarrow$ GO TO 429} \\
\hline Will probably buy & शायद खरीदेंगें & 04 & \\
\hline May or may not buy & $\begin{array}{r}\text { खरीद भी सकते है या } \\
\text { नही भी }\end{array}$ & 03 & \\
\hline Will probably not buy & शायद नही खरीदेंगें & 02 & \\
\hline Will definitely not buy & $\begin{array}{r}\text { निश्चित रूप से नही } \\
\text { खरीदेंगें }\end{array}$ & 01 & \multirow{2}{*}{$\begin{array}{l}\rightarrow \text { GO TO APPROACH } 1 \text { IF } \\
\text { CURRENT BUYER AND } \\
\text { APPROACH } 1 \text { NOT YET } \\
\text { DONE - ELSE GO TO Q501 }\end{array}$} \\
\hline Don't know (DO NOT SHOW) & पता नही & 98 & \\
\hline
\end{tabular}

\section{ASK Q429 IF CODED ‘5'l'4'l'3'l'2' IN Q428}

Q429 How many packs of [CONTROL PACK] would you buy in a week? RECORD VERBATIM WITH LEADING ZEROES आप एक सप्ताह मे के कितने पैक्स को खरीदेंगें ?

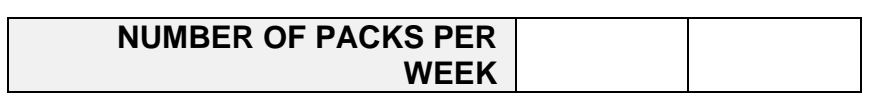

Q430 How many servings of [CONTROL PACK] would you give to [REFERENT CHILD] in a week? RECORD VERBATIM WITH LEADING ZEROES आप एक सप्ताह मे ............. को ............ की कितनी सर्विंग देंगें ?

\begin{tabular}{r|r|r|}
\hline NUMBER OF & $1621-22$ \\
SERVINGS PER WEEK & & \\
\hline
\end{tabular}

ASK Q431 IF CODED ‘9’ IN Q422

Q431 Would you feed [CONTROL PACK] to [REFERENT CHILD] if you would receive [CONTROL PACK] for FREE? SINGLE CODING ONLY

अगर आप को मुफत मे मिले तो क्या आप को यह देंगें ?

\begin{tabular}{|l|c|}
\hline $\begin{array}{l}\text { Yes } \\
\text { हाँ }\end{array}$ & 01 \\
\hline $\begin{array}{l}\text { No } \\
\text { नही }\end{array}$ & 02 \\
\hline $\begin{array}{l}\text { Don't know (DO NOT SHOW) } \\
\text { पता नही }\end{array}$ & 98 \\
\hline
\end{tabular}




\section{$\rightarrow$ ELSE GO Q501}

\section{ATTITUDES SECTION}

Q501 Did you receive any advice on which type of foods/ drinks you should give to your child? SINGLE CODING ONLY क्या आपने कोई सलाह प्राप्त की थी कि आपके बच्चे के लिए किस प्रकार का आहार/ ड्रिंक्स दिए जाने चाहिए ?

\begin{tabular}{|r|c|}
\hline $\begin{array}{r}\text { Yes } \\
\text { हाँ }\end{array}$ & 1 \\
\hline No & 2 \\
नही & 2 \\
\hline
\end{tabular}

Q502 SHOWCARD Q502 Do you remember who gave you the advice? MULTIPLE CODING POSSIBLE क्या आपको याद है सलाह किसने दी थी ?

\begin{tabular}{|c|c|c|}
\hline A paediatrician & एक बाल विशेषज्ञ & 01 \\
\hline Another type of health professional & स्वास्थ्य पेशेवर का कोई प्रकार & 02 \\
\hline A relative (own mom, aunt, mother-in-law) or friend & एक रिश्तेदार या दोस्त & 03 \\
\hline $\begin{array}{r}\text { Information from TV, radio, newspaper or other } \\
\text { media }\end{array}$ & $\begin{array}{l}\text { टीवी, रेडियो, अख्बार, या अन्य } \\
\text { मीडिया से जानकारी }\end{array}$ & 04 \\
\hline Others (Please specify & अन्य (कृप्या स्पष्ट करे ...........) & \\
\hline Others (Please specify & अन्य (कृप्या स्पष्ट करे ...........) & \\
\hline $\begin{array}{r}\text { Don't know/Don't remember (DON'T SHOWI } \\
\text { READ) }\end{array}$ & पता नही/ याद नही & 98 \\
\hline
\end{tabular}

Q504 Are there any foods containing iron? SINGLE CODING ONLY क्या किसी फूड/आहार में आयरन होता है ?

\begin{tabular}{|r|c|}
\hline Yes हाँ & 01 \\
\hline No नही & 02 \\
\hline $\begin{array}{r}\text { Don't know/Don't remember (DON'T SHOWI READ) } \\
\text { पता नही/ याद नही }\end{array}$ & 98 \\
\hline \multicolumn{2}{|c|}{$1647-48$}
\end{tabular}


ASK Q505I Q506 IF CODED '1' IN Q504

Q505 SHOWCARD Q505 Do you know which foods and drinks are rich in iron? MULTIPLE CODING POSSIBLE क्या आपको पता है कौन से खाद्य और पेय आयरन मे समृद्ध है ?

\begin{tabular}{|c|c|c|}
\hline Breast Milk & माँ का दूध & 01 \\
\hline $\begin{array}{l}\text { Cereals like Daliya/ Khichdi/ Mixed Grains/ } \\
\text { Sewiya }\end{array}$ & $\begin{array}{l}\text { सीरियल्स जैसे दलिया/खिचड़ी/मिले } \\
\text { अनाज/सेवईंया }\end{array}$ & 02 \\
\hline Cereals like Ragi/ Barleyl Bajra/ Maize/ Corn & $\begin{array}{l}\text { सीरियल्स जैसे } \\
\text { रागी/जों/बाजरा/मक्का/दाना }\end{array}$ & 03 \\
\hline Oats/ Corn flakes/ Rice flakes & ओट्स/ कोर्नफ्लेक्स/ चावल के फ्लेक्स & 04 \\
\hline Rice/ Rice based dishes & चावल/ चावल से बने व्यंजन & 05 \\
\hline Packaged Infant Cereals & पैकेज्ड इनफेंट सीरियल्स/ बेबी फूड & 06 \\
\hline Legumes like pulses, chana etc. & फली जैसे दालें, चना आदि & 07 \\
\hline Roti, Paratha, Bread, Dosa, Poori etc. & रोटी, पराठा, ब्रेड, डोसा, पूरी आदि & 08 \\
\hline Packaged Powdered Milk & पैकेज्ड पाउडर्ड दूध & 09 \\
\hline $\begin{array}{l}\text { Homemade Snacks like poha, semolina/ suji/ } \\
\text { rawa, halwa, upma, idli, puffed rice, Idiyappam, } \\
\text { sattu, chattuah, sabudana etc. }\end{array}$ & $\begin{array}{l}\text { घर में बने स्नेक्स जैसे पोहा, } \\
\text { सेमोलिना/सूजी/खा, हलवा, उपमा, } \\
\text { इडली, फूले चावल, इडियापन, सत्तू, } \\
\text { छतूआ, साबुदाना आदि }\end{array}$ & 10 \\
\hline Cow milk/ buffalo milk (packaged or loose) & $\begin{array}{l}\text { गाय का दूध/भैंस का दूध (पैकेज्ड या } \\
\text { खुला) }\end{array}$ & 11 \\
\hline $\begin{array}{l}\text { Milk products like curd, raita, lassi/ chaach, } \\
\text { cream, tea, coffee, paneer, khoya, shakes, ice } \\
\text { cream etc }\end{array}$ & $\begin{array}{l}\text { दूध के प्रोडक्ट्स जैसे दही, रायता, } \\
\text { लस्सी/छाछ, क्रीम, चाय, कॉफी, पनीर, } \\
\text { खोवा, शेक्स, आईसक्रीम आदि. }\end{array}$ & 12 \\
\hline Non vegetarian foods and non veg soups & माँसाहारी खाने और माँसाहारी सूप्स & 13 \\
\hline Infant formulas & इनफेंट फार्मूला & 14 \\
\hline Fruits/ Fruit Juices/ Neembu pani & फल/ फलों के जूस/ नींबू पानी & 15 \\
\hline $\begin{array}{l}\text { Packaged snacks/ foods like biscuits, chips, } \\
\text { soft drinks, confectionary items, namkeens, milk } \\
\text { additives likes Horlicks etc. }\end{array}$ & $\begin{array}{l}\text { पैकेज्ड स्नेक्स/फूड्स जैसे विस्कुट्स, } \\
\text { चिप्स, सॉफ्ट ड्रिंक्स, कॉन्फेक्शनरी } \\
\text { साम्रियाँ, नमकींस, दूध एडीटिव्स जेसे } \\
\text { हॉरलिक्स आदि }\end{array}$ & 16 \\
\hline Vegetables and vegetable soups & सब्जियाँ और सब्जियों के सूप्स & 17 \\
\hline \multicolumn{3}{|l|}{$\begin{array}{l}\text { Any other } \\
\text { कोई अन्य }\end{array}$} \\
\hline \multicolumn{3}{|l|}{$\begin{array}{l}\text { Any other } \\
\text { कोई अन्य }\end{array}$} \\
\hline \multicolumn{2}{|l|}{$\begin{array}{l}\text { Don't know/Don't remember (DON'T SHOWI READ) } \\
\text { पता नहीं/याद नहीं }\end{array}$} & 98 \\
\hline
\end{tabular}

Q506 SHOWCARD Q506 What are the health consequences of too little iron in foods and drinks? MULTIPLE CODING POSSIBLE

खाने और पीने मे बहुत थोडे आयरन के स्वार्थ्य परिणाम क्या है ?

\begin{tabular}{|c|c|c|}
\hline Impact on eyesight & आँखों की दृष्टि पर प्रभाव & 05 \\
\hline Anemia/ Blood deficiency & एनिमिया/ रक्त की कमी & 01 \\
\hline Lethargy/ Weakness & सुस्ती/ क्मजोरी & 02 \\
\hline Skin Related problems like allergy, rashes & $\begin{array}{r}\text { त्वचा से सम्बन्धित समस्यायें जैसे एलर्जी, } \\
\text { चकत्ते/ रैश }\end{array}$ & 06 \\
\hline Hair related problems & बालों से सम्बन्धित समस्यायें & 07 \\
\hline Irritability & $\begin{array}{l}\text { चिडचिडापन } \\
\end{array}$ & 03 \\
\hline Problems in digestion & पाचन की समस्यायें & 08 \\
\hline Impact on bones strength & हड्डियों की मजबूती पर प्रभाव & 09 \\
\hline Mental development & मानसिक विकास & 04 \\
\hline Others, please specify: & अन्य, कृप्या स्पष्ट करे & \\
\hline Others, please specify: & अन्य, कृप्या स्पष्ट करे & \\
\hline $\begin{array}{r}\text { Don't know/Don't remember (DON'T SHOWI } \\
\text { READ) }\end{array}$ & पता नही/ याद नही & 98 \\
\hline
\end{tabular}

\section{ASK Q510-514 IF PACKAGED INFANT CEREAL CODED IN Q301 (SERVED IN PAST 3 DAYS)}

Q510 Do you serve packaged infant cereals/ baby foods that contain added vitamins and minerals? SINGLE CODING ONLY क्या आप पैकेज्ड शिशु अनाज/ शिशु आहार सर्व करती है जो कि विटामिन और मिनरल युक्त होते है ? 


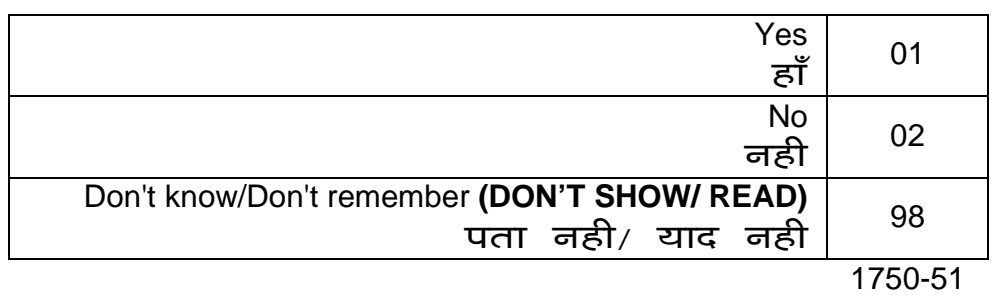

Q511 Until what age do you plan to provide packaged infant cereals/ baby foods to your child? IF DON'T KNOW; RECORD 98

किस उम्र तक आप आपके बच्चे के लिए पैकेज्ड शिशु अनाज/ शिशु आहार प्रदान करने की योजना बना रही है ?

\begin{tabular}{|l|l|l|l|}
\hline Maximum age of child (in years) & & \\
\hline
\end{tabular}

Q512 SHOWCARD Q512 Did you receive any advice on giving packaged infant cereals/ baby foods to [REFERENT CHILD]? SINGLE CODING ONLY

क्या आपने ........... के लिए पैकेज्ड शिशु अनाज/ शिशु आहार देने पर कोई सलाह प्राप्त की थी ?

\begin{tabular}{|r|r|r|}
\hline $\begin{array}{r}\text { Yes, I have received advice NOT to give } \\
\text { packaged infant cereals/ baby foods to } \\
\text { [REFERENT CHILD] }\end{array}$ & $\begin{array}{r}\text { हाँ, मैने ....... के लिए पैकेज्ड शिशु } \\
\text { अनाज/ शिशु आहार नही देने की } \\
\text { सलाह प्राप्त की है }\end{array}$ & 01 \\
\hline $\begin{array}{r}\text { Yes, I have received advice to give } \\
\text { packaged infant cereals/ baby foods to } \\
\text { [REFERENT CHILD] }\end{array}$ & $\begin{array}{c}\text { हाँ, मैने } \ldots . . . . \text { के लिए पैकेज्ड शिशु } \\
\text { अनाज/ शिशु आहार देने की सलाह } \\
\text { प्राप्त की है }\end{array}$ & 02 \\
\hline No, I have not received any advice & $\begin{array}{r}\text { नही, मैने कोई सलाह प्राप्त नही की } \\
\text { है }\end{array}$ & 03 \\
\hline $\begin{array}{r}\text { Don't know/Don't remember (DON'T SHOWI } \\
\text { READ) }\end{array}$ & पता नही/ याद नही & 98 \\
\hline
\end{tabular}

Q513 SHOWCARD Q513 Do you remember who gave you the advice? MULTIPLE CODING POSSIBLE क्या आपको याद है सलाह किसने दी थी ?

\begin{tabular}{|c|c|c|}
\hline A paediatrician & एक बाल विशेषज्ञ & 01 \\
\hline Another type of health professional & स्वास्थ्य पेशेवर का कोई प्रकार & 02 \\
\hline A midwife & एक मिडवाइफ & 03 \\
\hline $\begin{array}{r}\text { A relative (own mom, aunt, mother-in- } \\
\text { law) or friend }\end{array}$ & एक रिश्तेदार या दोस्त & 04 \\
\hline $\begin{array}{r}\text { Information from TV, radio, newspaper or } \\
\text { other media }\end{array}$ & $\begin{array}{l}\text { टीवी, रेडियो, अख्बार, या अन्य मीडिया से } \\
\text { जानकारी }\end{array}$ & 05 \\
\hline Others, please specify: & अन्य, कृप्या स्पष्ट करे & \\
\hline $\begin{array}{r}\text { Don't know/Don't remember } \\
\text { (DON'T SHOW/ READ) }\end{array}$ & पता नही/ याद नही & 98 \\
\hline
\end{tabular}

Q514 What was the age of [REFERENT CHILD] when the advice was given? RECORD IN MONTHS. IF ADVICE WAS GIVEN DURING PREGNANCY PUT "00"

............... की उम्र क्या थी जब सलाह गयी थी ?

Age of child (IN MONTHS) when advice was given

1766-67

\section{CLASSIFICATION SECTION}

Q601 How many people are permanently living in this house? Please exclude household help/s if any. RECORD VERBATIM WITH LEADING ZEROES

इस परिवार मे स्थायी रूप से कितने लोग रहते है ? कृप्या घरेलु सहायको को शामिल नही करे ।

No. of members in $\mathrm{HH}$

\begin{tabular}{|l|l|l|}
\hline No. of members in $\mathbf{H H}$ & & \\
\hline
\end{tabular}

$1768-69$

Q602 And how many working members are there in your household? RECORD VERBATIM WITH LEADING ZEROES और आपके परिवार मे कितने कार्यरत सदस्य है ?

\begin{tabular}{|l|l|l|} 
No. of working members in $\mathbf{H H}$ & & \\
1770-71
\end{tabular}


Q603 May we know which of these monthly household income ranges does your household monthly income belongs to? SINGLE CODING ONLY

क्या हम जान सकते है इनमे से कौन सा मासिक पारिवारीक आय रेंज मे आपके परिवार की मासिक आय संबंध रखती है ?

\begin{tabular}{|l|c|}
\hline $\begin{array}{l}\text { Less than Rs. 3,000 } \\
\text { 3 0 0 0 रूपए से कम }\end{array}$ & 1 \\
\hline Rs. 3,001 to Rs. 5,000 & 2 \\
\hline Rs. 5,001 to Rs. 10,000 & 3 \\
\hline Rs. 10,001 to Rs. 20,000 & 4 \\
\hline Rs. 20,001 to Rs. 30,000 & 5 \\
\hline Rs. 30,001 to Rs. 40,000 & 6 \\
\hline Rs. 40,001 to Rs. 50,000 & 7 \\
\hline $\begin{array}{l}\text { More than Rs. 50,000 } \\
\text { 5 0, 0 0 रूपए से ज्यादा }\end{array}$ & 8 \\
\hline $\begin{array}{l}\text { Not disclosed (DO NOT SHOWI READ) } \\
\text { नही बताया }\end{array}$ & 9 \\
\hline
\end{tabular}

Q604 SHOWCARD Q604 Please tell me which of the following applies to the your house? SINGLE CODING ONLY कृप्या मुझो बताए निम्नलिखित मे से कौन सा आपके घर के लिए लागु होता है ?

\begin{tabular}{|l|l|c|}
\hline Apartment & अपार्टमेंट & 1 \\
\hline Bungalow & बंग्लो & 2 \\
\hline Independent House & स्वतंत्र मकान & 3 \\
\hline Independent Floor & स्वतंत्र फ्लोर & 4 \\
\hline
\end{tabular}

Q605 SHOWCARD Q605 And which of the following applies to the ownership of this house? SINGLE CODING ONLY और निम्नलिखित मे से कौन सा इस घर के लिए स्वामित्व पर लागु होता है ?

\begin{tabular}{|l|l|l|}
\hline Rented & किराए का & 1 \\
\hline Owned & $\begin{array}{l}\text { स्वामित्व } \\
\text { प्राप्त }\end{array}$ & 2 \\
\hline
\end{tabular}

Q606 Do you or any member in your household own any other house? SINGLE CODING ONLY क्या आप या आपके परिवार का कोई सदस्य किसी अन्य घर का स्वामित्व रखता है ?

Q607 Do you or any member in your household own any agricultural land? SINGLE CODING ONLY क्या आप या आपके परिवार का कोई सदस्य किसी कृषि योग्य भुमि का स्वामित्व रखता है ?

Q608 Do you or any member in your household have a bank account or a post office account? SINGLE CODING ONLY क्या आप या आपके परिवार के किसी सदस्य के पास बेंक खाता या डाक घर खाता है ?

\begin{tabular}{|l|c|c|c|}
\hline & Q606 & Q607 & Q608 \\
\hline Yes हाँ & 1 & 1 & 1 \\
\hline No नही & 2 & 2 & 2 \\
\hline
\end{tabular}


Q609 SHOW CARD Q609 what is the main source of drinking water for members of your household? SINGLE CODING ONLY

आपके परिवार के सदस्यों के लिए पीने के पानी का मुख्य सोत क्या है ?

Q610 SHOW CARD Q610 what is the main source of main source of water used by members of your household for other purposes such as cooking and hand washing? SINGLE CODING ONLY

आपके परिवार के सदस्यों द्वारा अन्य उद्देश्यो जैसे कूकिंग और हाथ धोने के लिए इस्तेमाल किए जाने वाले पानी के मुख्य सोत क्या है ?

\begin{tabular}{|c|c|c|c|}
\hline & & Q609 & Q610 \\
\hline Piped water & पाईप वाला पानी & & \\
\hline Piped to dwelling & घर में पाईप से & 01 & 01 \\
\hline Piped to yard/plot & यार्ड/प्लॉट में पाईप से & 02 & 02 \\
\hline From public tap, standpipe & पब्लिक नल/स्टेंडपाईप से & 03 & 03 \\
\hline Water from tube well or borehole & नलकूप या बोरवेल से पानी & 04 & 04 \\
\hline Rainwater & बरसात का पानी & 05 & 05 \\
\hline Dug well & खुदे हुए कुएँ & & \\
\hline Protected well & सुरक्षित कुआँ & 06 & 06 \\
\hline Unprotected well & असुरक्षित कुआँ & 07 & 07 \\
\hline Water from spring & झरने का पानी & & \\
\hline Protected spring & सुरक्षित झरना & 08 & 08 \\
\hline Unprotected spring & असुरक्षित झरना & 09 & 09 \\
\hline Tanker truck & टेंकर ट्रक & 10 & 10 \\
\hline Cart with small tank & ठेले वाला छोटा टेंक & 11 & 11 \\
\hline $\begin{array}{l}\text { Surface water (river/dam/ } \\
\text { lake/pond/stream/canal/irrigation channel) }\end{array}$ & $\begin{array}{l}\text { सतह का पानी (नदी/ डेम/ झील/ } \\
\text { तालाब/भाप/नहर/सिंचाई चैनल) }\end{array}$ & 12 & 12 \\
\hline Bottled water & बोतल का पानी & 13 & 13 \\
\hline $\begin{array}{l}\text { Other (please specify) } \\
\text { अन्य (कृप्या स्पष्ट करे) }\end{array}$ & & & \\
\hline
\end{tabular}

Q611 Do you do anything to the water to make it safer to drink? SINGLE CODING ONLY

क्या आप पानी को सुरक्षित पीने का पानी बनाने के लिए कुछ करते है ?

\begin{tabular}{|l|c|l|}
\hline $\begin{array}{l}\text { Yes } \\
\text { हाँ }\end{array}$ & 1 & $\rightarrow$ GO TO 612 \\
\hline $\begin{array}{l}\text { No } \\
\text { नही }\end{array}$ & 2 & $\rightarrow$ GO TO 613 \\
\hline \multicolumn{3}{|c|}{1782} \\
\hline
\end{tabular}

\section{ASK IF '1' CODED IN Q611}

Q612 SHOW CARD Q612 Please tell me what do you do to make the water safer to drink? SINGLE CODING ONLY कृप्या मुझे बताए आप पानी को सुरक्षित पीने का पानी बनाने के लिए क्या करते है ?

\begin{tabular}{|l|l|c|}
\hline Boil it & उसे उबालते है & 1 \\
\hline Filter it using water filter & $\begin{array}{l}\text { वॉटर फिल्टर का उपयोग करके उसे } \\
\text { फिल्टर करते है }\end{array}$ & 2 \\
\hline Use water purifier/ RO & $\begin{array}{l}\text { वॉटर प्युरीफायर/ आर ओ का इस्तेमाल } \\
\text { करते है }\end{array}$ & 3 \\
\hline Any other (please specify) & कोई अन्य (कृप्या स्पष्ट करे) & 1783 \\
\hline
\end{tabular}

Q613 Do you use a different water source for your children? SINGLE CODING ONLY

क्या आपके बच्चो के लिए आप अलग अलग पानी के सोत उपयोग करते है ?

\begin{tabular}{|l|c|l|}
\hline $\begin{array}{l}\text { Yes } \\
\text { हाँ }\end{array}$ & 1 & $\rightarrow$ GO TO 614 \\
\hline $\begin{array}{l}\text { No } \\
\text { नही }\end{array}$ & 2 & $\rightarrow$ GO TO 615 \\
\hline
\end{tabular}


ASK IF '1' CODED IN Q613

Q614 SHOW CARD Q614 Which water source do you use for your children? SINGLE CODING ONLY आपके बच्चो के लिए आप कौन सा पानी का सोत इस्तेमाल करते है ?

\begin{tabular}{|c|c|c|}
\hline Piped water & पाईप वाला पानी & \\
\hline Piped to dwelling & घर में पाईप से & 01 \\
\hline Piped to yard/plot & यार्ड/प्लॉट कें पाईप से & 02 \\
\hline From public tap, standpipe & पब्लिक नल/स्टेंडपाईप से & 03 \\
\hline Water from tube well or borehole & नलकूप या बोरबेल से पानी & 04 \\
\hline Rainwater & बरसात का पानी & 05 \\
\hline Dug well & खुदे हुए कुएँ & \\
\hline Protected well & सुरक्षित कुआँ & 06 \\
\hline Unprotected well & असुरक्षित कुआँ & 07 \\
\hline Water from spring & झरने का पानी & \\
\hline Protected spring & सुरक्षित झरना & 08 \\
\hline Unprotected spring & असुरक्षित झरना & 09 \\
\hline Tanker truck & टेंकर ट्रक & 10 \\
\hline Cart with small tank & ठेले वाला छोटा टेंक & 11 \\
\hline $\begin{array}{l}\text { Surface water (river/dam/ } \\
\text { lake/pond/stream/canal/irrigation channel) }\end{array}$ & $\begin{array}{l}\text { सतह का पानी (नदी/ डेम/ झील/ } \\
\text { तालाब/भाप/नहर/सिंचाई चेनल) }\end{array}$ & 12 \\
\hline Bottled water & बोतल का पानी & 13 \\
\hline $\begin{array}{l}\text { Other (please specify) } \\
\text { अन्य (कृप्या स्पष्ट करे) }\end{array}$ & & \\
\hline
\end{tabular}

Q615 Do you do anything to the water to make water safer for your children to drink? SINGLE CODING ONLY क्या आप पानी को सुरक्षित पीने का पानी बनाने के लिए कुछ करते है ?

\begin{tabular}{|l|c|l|}
\hline Yes हाँ & 1 & $\rightarrow$ GO TO 616 \\
\hline No नही & 2 & $\rightarrow$ GO TO 617 \\
\hline
\end{tabular}

ASK IF '1' CODED IN Q615

Q616 SHOW CARD Q616 Please tell me what do you do to make the water for your children safer to drink? SINGLE CODING ONLY

कृप्या मुझे बताए आपके बच्चे के लिए पीने के पानी को सुरक्षित बनाने के लिए आप क्या करते है ?

\begin{tabular}{|l|l|c|}
\hline Boil it & उसे उबालते है & 1 \\
\hline Filter it using water filter & $\begin{array}{l}\text { वॉटर फिल्टर का उपयोग करके उसे } \\
\text { फिल्टर करते है }\end{array}$ & 2 \\
\hline Use water purifier/ RO & $\begin{array}{l}\text { वॉटर प्युरीफायर/ आरओ का इस्तेमाल } \\
\text { करते है }\end{array}$ & 3 \\
\hline Any other (please specify) & कोई अन्य (कृप्या स्पष्ट करे) & \\
\hline
\end{tabular}

Q617 SHOW CARD Q617 What type of fuel does your household mainly use for cooking? SINGLE CODING ONLY आपके परिवार मे खाना पकाने के लिए मुख्य रूप से किस प्रकार का ईधन उपयोग किया जाता है ?

\begin{tabular}{|l|l|l|}
\hline Electricity & बिजली & 01 \\
\hline LPG/ Natural Gas & एलपीजी/ प्राकृतिक गैस & 02 \\
\hline Biogas & बायोगैस & 03 \\
\hline Kerosene & मिट्टी का तेल & 04 \\
\hline Coal/ Lignite & कोयला/ लिगनाइट & 05 \\
\hline Charcoal & चारकोल & 06 \\
\hline Wood & लकडी & 07 \\
\hline Straws/ shrubs/grass & घास/ भुसा/ तिनके & 08 \\
\hline Agricultural crop waste & कृषि फसल का कचरा & 09 \\
\hline Dung Cakes & गोबर के उपले & 10 \\
\hline Any Other & कोई अन्य & \\
\hline
\end{tabular}

\section{SHOWCARD Q618}

Q618 Please look at this card and tell me what kind of toilet facility do members of your household usually use? SINGLE CODING ONLY

कृप्या इस कार्ड को देखे और मुझे बताए आपका परिवार आमतोर पर किस प्रकार की शौचालय सुविधा का इस्तेमाल कर रहा है ?

\begin{tabular}{|c|l|c|}
\hline FLUSH OR POUR FLUSH TOILET & $\begin{array}{l}\text { फ्लश या पानी डालने वाला फ्लश } \\
\text { टॉयलेट }\end{array}$ \\
\hline Flush to piped sewer system & पाईप सीवर सिस्टम वाला फ्लश & 01 \\
\hline
\end{tabular}




\begin{tabular}{|c|c|c|}
\hline Flush to septic tank & सेप्टिक टेंक वाला फ्लश & 02 \\
\hline Flush to pit latrine & पिट लैट्रीन वाला फ्लश & 03 \\
\hline Flush to somewhere else & कहीं और फ्लश & 04 \\
\hline Flush, don't know where & फ्लश कहाँ है नहीं जानता & 05 \\
\hline PIT LATRINE & पिट लैट्रीन & \\
\hline Ventilated improved pit (VIP) / biogas latrine & $\begin{array}{l}\text { वेनटीलेटेड इम्प्रूट्ड पिट } \\
\text { (वीआईपी)/बॉयोगैस लेट्रीन }\end{array}$ & 06 \\
\hline Pit latrine with slab & स्लेब वाली पिट लैट्रीन & 07 \\
\hline Pit latrine without slab/ Open pit & $\begin{array}{l}\text { बिना स्लेब वाली पिट लैट्रीन/ खुला } \\
\text { पिट/ गढ्ठा }\end{array}$ & 08 \\
\hline Twin pit/composting toilet & ट्वीन/दो पिट/ कम्पोजिटिंग टॉयलेट & 09 \\
\hline Dry toilet & ड्राई टॉयलेट & 10 \\
\hline No facility/uses open space Or field & $\begin{array}{l}\text { कोई सुविधा नहीं/ खुली जगहों या } \\
\text { मैदान का इस्तममाल करते हैं }\end{array}$ & 11 \\
\hline $\begin{array}{l}\text { Any Other } \\
\text { कोई अन्य }\end{array}$ & & \\
\hline
\end{tabular}

Q619 And how many toilets are there in your household? RECORD VERBATIM WITH LEADING ZEROES और आपके परिवार मे कितने शौचालय है ?

\begin{tabular}{|c|l|}
$\begin{array}{c}\text { No. of toilets in HH } \\
\text { परिवार मे शौचालयो की }\end{array}$ & \\
संख्या &
\end{tabular}

Q620 Do you share this toilet facility with other households? SINGLE CODING ONLY

क्या आप अन्य परिवारो के साथ इस टॉयलेट सुविधा को बांटते है ?

\begin{tabular}{|l|c|}
\hline Yes हाँ & 1 \\
\hline No नहीं & 2 \\
\hline \multicolumn{2}{|c|}{1817} \\
\hline
\end{tabular}

Q621 SHOWCARD Q621 Please look at this card and tell me, does your household or any member of your household have/own: MULTIPLE CODING POSSIBLE

कृप्या इस कार्ड को देखे और मुझे बताए, क्या आपके परिवार या परिवार के किसी सदस्य के पास ....... का स्वामित्व है।

\begin{tabular}{|c|c|c|c|c|c|}
\hline Electricity Connection & $\begin{array}{l}\text { बिजली का } \\
\text { कनेक्शन }\end{array}$ & 01 & Motorcycle / scooter & मोटरसाइकिल/ स्कूटर & 16 \\
\hline Mattress & मैट्रस & 02 & Refrigerator & रेफीजरेटर & 17 \\
\hline Pressure cooker & प्रेशर कूकर & 03 & Mobile phone & टेलीफोन/ मोबाइल & 18 \\
\hline Chair & कुर्सी & 04 & Any other type of phone & $\begin{array}{l}\text { कोई अन्य प्रकार का } \\
\text { फोन }\end{array}$ & 19 \\
\hline Cot or bed & कोट या बिस्तर & 05 & B/W Television & ब्लैक/व्हाइट टेलीविजन & 20 \\
\hline Table & टेबल & 06 & Color Television & कलर टेलीविजन & 21 \\
\hline Sewing machine & सिलाई मशीन & 07 & Washing Machine & वॉशिंग मशीन & 22 \\
\hline Watch/ clock & घडी & 08 & CD/ VCD/ DVD player & $\begin{array}{l}\text { सीडी/ वीसीडी/ डीवीडी } \\
\text { प्लेयर }\end{array}$ & 23 \\
\hline Bicycle & साइकिल & 09 & Radio or transistor & रेडियो या ट्रंसिस्टर & 24 \\
\hline Animal drawn cart & $\begin{array}{l}\text { जानवर द्वारा } \\
\text { चलने वाली गाडी }\end{array}$ & 10 & $\begin{array}{l}\text { Personal computer/ } \\
\text { laptop }\end{array}$ & $\begin{array}{l}\text { पर्सनल कंप्यूटर/ } \\
\text { लैपटॉप }\end{array}$ & 25 \\
\hline Water pump & पानी का पम्प & 11 & Car & कार & 26 \\
\hline Tractor & ट्रैक्टर & 12 & Truck/ jeep/ van & ट्रक/ जीप/ वैन & 27 \\
\hline Electric fan & विजली का पंखा & 13 & Air conditioner & एयर कंडीश्नर & 28 \\
\hline LPG stove & एलपीजी स्टोव & 14 & Threshers & श्रेशर्स & 29 \\
\hline Stove with electricity & $\begin{array}{l}\text { बिजली के साथ } \\
\text { स्टोव }\end{array}$ & 15 & & & \\
\hline
\end{tabular}

$1818-67$

Q622 How many rooms in this household are used for sleeping? RECORD VERBATIM WITH LEADING ZEROES इस परिवार मे कितने कमरो को सोने के लिए इस्तेमाल किया जाता है ?

\begin{tabular}{|l|l|}
\hline परिवार मे कितने कमरो को सोने के लिए इस्तेमाल किया जाता है ? \\
No. of rooms used for sleeping in HH & \\
परिवार मे सोने के लिए इस्तेमाल होने वाले \\
कमरो की संख्या
\end{tabular}

Q623 Main material of the floor. RECORD OBSERVATION फर्श की मुख्य सामग्री

\begin{tabular}{|c|c|c|}
\hline Natural Floor & प्राकृतिक जमीन & \\
\hline Mud / clay/ earth & गीली मिट्टी/ चिकनी मिट्टी & 01 \\
\hline
\end{tabular}




\begin{tabular}{|c|c|c|}
\hline Sand & रेत & 02 \\
\hline Dung & गोबर & 03 \\
\hline Rudimentary Floor & अल्पविकसित जमीन & \\
\hline Raw Wood planks & कच्ची लकड़ी का तख्ता & 04 \\
\hline Palm/ Bamboo & ताड़/बांस & 05 \\
\hline Brick & ईंट & 06 \\
\hline Stone & पत्थर & 07 \\
\hline Finished floor & तैयार जमीन & \\
\hline Parquet or polished wood & काठ-खण्ड या पॉलिश लकड़ी & 08 \\
\hline Vinyl or asphalt & प्लास्टिक या डामर & 09 \\
\hline Ceramic tiles & सेरामिक टाईल्स & 10 \\
\hline Cement & सीमेंट & 11 \\
\hline Carpet & कॉरपेट & 12 \\
\hline Polished stone/marble/Granite & $\begin{array}{l}\text { पालिश किया हुआ पत्थर/ मारबल/ } \\
\text { ग्रेनाईट }\end{array}$ & 13 \\
\hline $\begin{array}{l}\text { Any Other } \\
\text { कोई } \\
\text { अन्य }\end{array}$ & & \\
\hline
\end{tabular}

Q624 Main material of the roof. RECORD OBSERVATION

छत की मुख्य सामग्री।

\begin{tabular}{|c|c|c|}
\hline NATURAL ROOFING & प्राकृतिक छत & \\
\hline No roof & कोई छत नहीं & 01 \\
\hline Thatch/palm leaf/reed/grass & $\begin{array}{l}\text { छप्पर/ताड़ के पत्ते/सरकण्डा/ } \\
\text { घास }\end{array}$ & 02 \\
\hline Mud & गीली मिट्टी & 03 \\
\hline Sod/mud and grass mixture & $\begin{array}{l}\text { घास/गीली मिट्टी और घास का } \\
\text { मिश्रण }\end{array}$ & 04 \\
\hline Plastic/polythene sheeting & प्लास्टिक/पॉलीथीन शीटिग & 05 \\
\hline RUDIMENTARY ROOFING & अल्पविकसित छत & \\
\hline Rustic mat & साधारण चटाई & 06 \\
\hline Palm/bamboo & ताड़/बांस & 07 \\
\hline Raw wood planks/timber & कच्ची लकड़ी का तख्ता/ टिम्बर & 08 \\
\hline Unburnt brick & बिना जली ईंट & 09 \\
\hline Loosely packed stone & ढीले पैक्ड पत्थर & 10 \\
\hline FINISHED ROOFING & तैयार छत & \\
\hline Metal/gi (galvanized iron) & मेटल/जी आई & 11 \\
\hline Wood & लकड़ी & 12 \\
\hline Calamine/cement fiber & केलेमाईन/ सीमेंट फाईबर & 13 \\
\hline Asbestos sheets & ऐस्वेस्टस शीट्स & 14 \\
\hline Rcc/rbc/cement/concrete & $\begin{array}{l}\text { आरसीसी/ आरबीसी/ सीमेंट/ } \\
\text { कंक्रीट }\end{array}$ & 15 \\
\hline Roofing shingles & रूफिग शिंगल्स & 16 \\
\hline Tiles & टाईल्स & 17 \\
\hline Slate & स्लेट & 18 \\
\hline Burnt brick & जली इंट & 19 \\
\hline $\begin{array}{l}\text { Any Other } \\
\text { कोई } \\
\text { अन्य }\end{array}$ & & \\
\hline
\end{tabular}

Q625 Main material of the walls. RECORD OBSERVATION

दीवारो की मुख्य सामग्री।

\begin{tabular}{|c|c|c|}
\hline NATURAL WALLS & प्राकृतिक दीवारें & 01 \\
\hline No walls & कोई दीवारें नहीं & 02 \\
\hline Cane/palm/trunks/bamboo & बेंत/ ताड़/ ट्रंक्स/ बांस & 03 \\
\hline Mud & गीली मिट्टी & 04 \\
\hline Grass/reeds/thatch & घास/ सरकण्डा/ छप्पर & 05 \\
\hline RUDIMENTARY WALLS & अल्पविकरित दीवारें & 06 \\
\hline Bamboo with mud & गीली मिट्टी के साथ बांस & 07 \\
\hline Stone with mud & गीली मिट्टी के साथ पत्थर & 08 \\
\hline Plywood & प्लाईवुड & 09 \\
\hline Cardboard & कार्डबोर्ड & 10 \\
\hline Unburnt brick & बिना जली ईंट & \\
\hline Raw wood/reused wood & कच्ची लकड़ी/ फिर से इस्तेमाल की & \\
\hline
\end{tabular}




\begin{tabular}{|l|l|l|}
\hline & गयी लकड़ी & \\
\hline FINISHED WALLS & तैयार दीवारें & 11 \\
\hline Cement/concrete & सीमेंट/कंक्रीट & 12 \\
\hline Stone with lime/cement & चूना/ सीमेंट के साथ पत्थर & 13 \\
\hline Burnt bricks & जली इंटें & 14 \\
\hline Cement blocks & सीमेंट ब्लोक्स & 15 \\
\hline Wood planks/shingles & लकड़ी का तख्ता/ शिंगल्स & 16 \\
\hline Gi/metal/asbestos sheets & जी आई/ मेटल/ ऐस्वेस्टर्स शीट्स & \\
\hline $\begin{array}{l}\text { Any Other } \\
\text { कोई } \\
\text { अन्य }\end{array}$ & & \\
\hline
\end{tabular}

Type of Windows. RECORD OBSERVATION

खिडकियों के प्रकार।

\begin{tabular}{|c|c|c|c|}
\hline & & YES & NO \\
\hline Windows with glass & शीशे के साथ खिडकियां & 1 & 2 \\
\hline Windows with screen & स्कीन के साथ खिडकियां & 1 & 2 \\
\hline Windows with curtains / shutters & पर्दो/ शटर्स के साथ खिडकियां & 1 & 2 \\
\hline Any Other & कोई अन्य & 1 & 2 \\
\hline
\end{tabular}

SAY: I would like to know something about the Chief Wage Earner in your household. By Chief Wage Earner I mean the person who contributes the most to the household income.

कहेः मै आपके परिवार मे मुख्य कमाने वाले सदस्य के बारे मे कुछ जानना चाहूंगा। मुख्य कमाने वाले द्वारा मेरा मतलब व्यक्ति जो पारिवारीक आय के लिए सबसे ज्यादा योगदान देता है।

Q627 What is his/her occupation (IF RETIRED, ASK: What was his/her occupation before he/she retired?) RECORD VERBATIM AND THEN CODE APPROPRIATELY IN GRID AFTER Q628.

उनका व्यवसाय क्या है (यदि रिटायर्ड, पूछे: रिटायर होने से पहले उनका व्यवसाय क्या था ?)

\section{RECORD VERBATIM:}

Q628 IF THE PERSON IS A BUSINESSMAN I INDUSTRIALIST, ASK "Roughly how many persons in all are employed by the CWE at his / her establishment?"

अंदाजन मुख्य कमाने वाले के व्यवसाय पर कितने लोग कार्यरत है ?

\section{\begin{tabular}{|l|l|l|l|l|}
\hline No. of employees: & & & & \\
\hline
\end{tabular}}

Q629 What is the highest level to which he/she has studied? RECORD VERBATIM AND THEN CODE APPROPRIATELY IN GRID BELOW THEN CODE SEC).

उनके द्वारा प्राप्त किया गया शिक्षा का उच्चतम स्तर क्या है ?

RECORD VERBATIM:

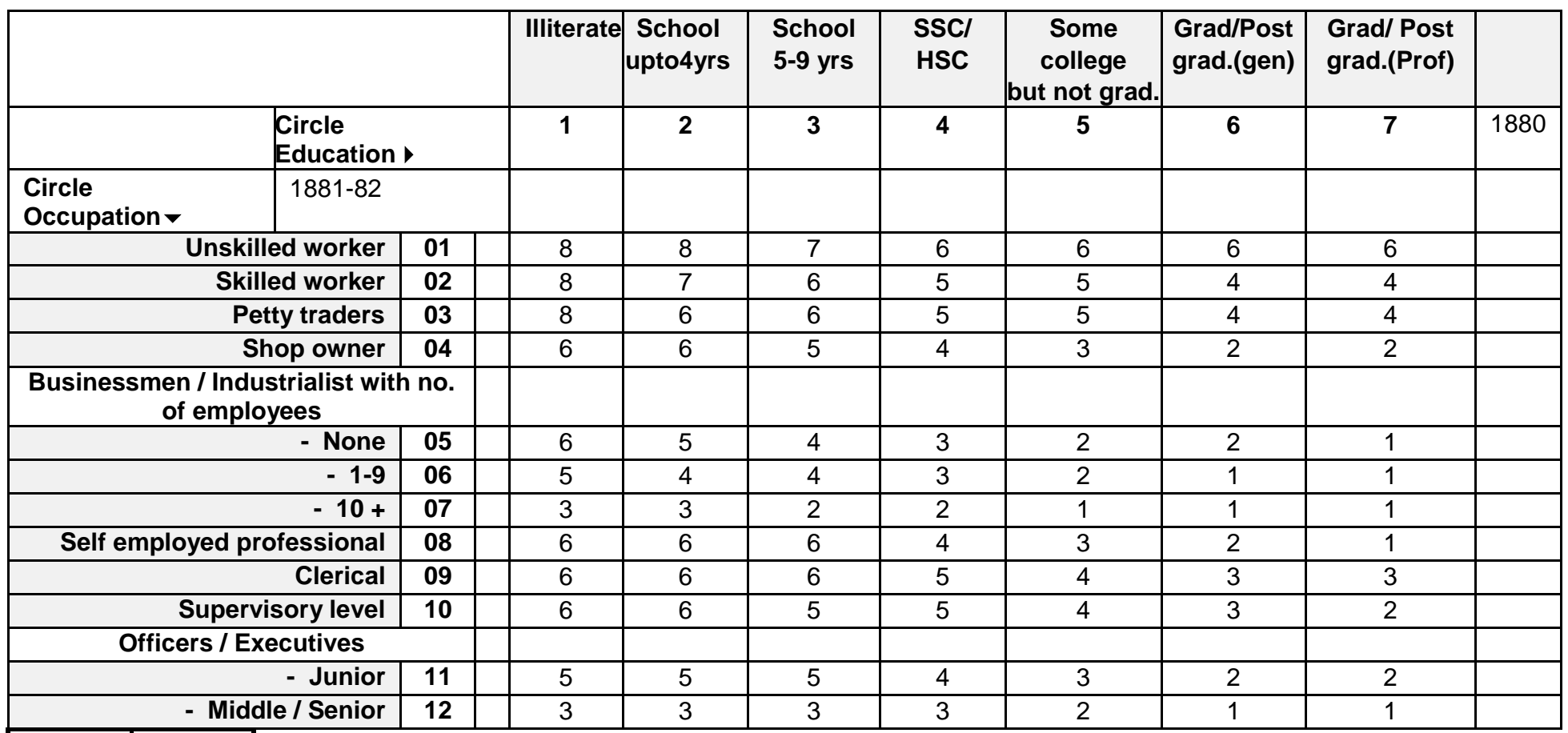

\begin{tabular}{l|l|l|l|l}
\hline SEC: & & 1883
\end{tabular}

Q630 SHOWCARD Q630 Please tell me which of the following applies to your household. SINGLE CODING ONLY कृपया मुझे बताये कि आपके घर पर इनमें से कौन-सा लागू होता है ? 


\begin{tabular}{|c|c|c|}
\hline No one eat non vegetarian food & कोई भी माँसाहारी खाना नहीं \\
खाता है & 1 \\
\hline Some members eat non vegetarian food & $\begin{array}{c}\text { कुछ सदस्य माँसाहारी खाना } \\
\text { खाते हैं }\end{array}$ & 2 \\
\hline All members eat non vegetarian food & $\begin{array}{c}\text { सभी सदस्य माँसाहारी खाना } \\
\text { खाते है }\end{array}$ & 3 \\
\hline
\end{tabular}

When REFERENT CHILD was last sick with diarrhea did you take him/ her to the doctor? SINGLE CODING ONLY जब सम्बन्धित बच्चा पिछली बार दस्त से बीमार था तो क्या आप उसे डॉक्टर के पास ले गर्यीं ?

Q632 How much did you spend for the treatment of diarrhea (consultation and medication cost)? RECORD VERBATIM WITH LEADING ZEROES

आपने दस्त के उपचार के लिए कितना खर्च किया (कंसलटेशन/परमर्श और दवाई के खर्च को मिलाकर) ?

\begin{tabular}{|r|c|c|r|l|l|}
\hline & Q631 & \multicolumn{3}{|c|}{ Q632 (in Rs.) } & \\
\hline $\begin{array}{r}\text { Yes } \\
\text { हाँ }\end{array}$ & 1 & & & & \\
\hline $\begin{array}{r}\text { No } \\
\text { नहीं }\end{array}$ & 2 & & & & \\
\hline $\begin{aligned} \text { Child did not suffer from this disease } \\
\text { बच्चे को यह बीमारी नहीं हुई }\end{aligned}$ & 3 & & & & \\
\hline
\end{tabular}

Q633 When REFERENT CHILD was last sick with lower respiratory tract infection (like bronchitis, pneumonia) did you take $\mathrm{him} /$ her to the doctor? SINGLE CODING ONLY

जब सम्बन्धित बच्चा पिछली बार लोवर रेसपिटेटरी ट्रेट इनफेक्शन (जैसे फेफड़े की सूजन, नीमोनिया) से बीमार था तो क्या आप उसे डॉक्टर के पास ले गर्यी ?

Q634 How much did you spend for the treatment of lower respiratory tract infection (like bronchitis, pneumonia)? RECORD VERBATIM WITH LEADING ZEROES

आपने लोवर रेसपिटेटरी ट्रेट इनफेक्शन (जैसे फेफड़े की सूजन, नीमोनिया) के उपचार पर कितना खर्च किया ?

\begin{tabular}{|c|c|c|}
\hline & Q633 & Q634 (in Rs.) \\
\hline $\begin{array}{l}\text { Yes } \\
\text { हाँ }\end{array}$ & 1 & \\
\hline $\begin{array}{r}\text { No } \\
\text { नहीं }\end{array}$ & 2 & \\
\hline $\begin{array}{l}\text { Child did not suffer from this disease } \\
\text { बच्चे को यह बीमारी नहीं हुई }\end{array}$ & 3 & \\
\hline
\end{tabular}


Q503 Now, l'll read out some statements in regard with nutritional requirements of kids in the age group 6-23 months. As I read each statement, please look at this card and tell me, SHOWCARD Q503 how strongly do you agree or disagree that this statement applies to you. READ OUT STATEMENTS ONE BY ONE. ROTATE STATEMENTS. SINGLE CODING PER STATEMENT

अब मे 6-23 महीनो के आयु समुह मे बच्चो की आवश्यक पोषक के संबंध मे कुछ वाक्यों को पढूंगा। जैसे मै प्रत्येक वाक्य पढूं, कृप्या इस कार्ड को देखे और मुझे बताए, आपके लिए यह वाक्य लागु होता इस बात से आप कितना सहमत या असहमत है।

\begin{tabular}{|c|c|c|c|c|c|c|c|}
\hline & TICK START & 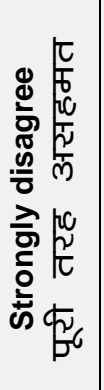 & 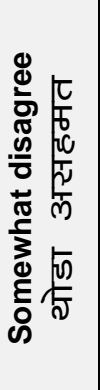 & 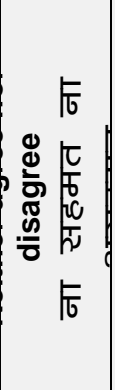 & 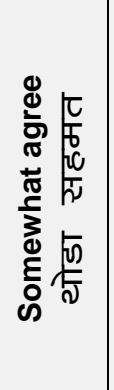 & 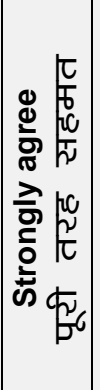 & 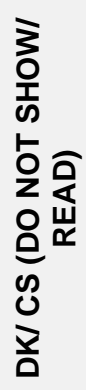 \\
\hline $\begin{array}{r}\text { Carbohydrates are } \\
\text { required to provide energy } \\
\text { to child }\end{array}$ & $\begin{array}{l}\text { बच्चे को उर्जा प्रदान करने के लिए } \\
\text { कार्बोहाइड्रेट आवश्यक है }\end{array}$ & 1 & 2 & 3 & 4 & 5 & 9 \\
\hline $\begin{array}{l}\text { Calcium is important for } \\
\text { bone development of child }\end{array}$ & $\begin{array}{l}\text { बच्चे के हड्डी के विकास के लिए } \\
\text { कैल्सियम महत्वपूर्ण है }\end{array}$ & 1 & 2 & 3 & 4 & 5 & 9 \\
\hline $\begin{array}{l}\text { Calcium is important for } \\
\text { tooth development of child }\end{array}$ & $\begin{array}{l}\text { बच्चे के दांतो के विकास के लिए } \\
\text { कैल्सियम महत्वपूर्ण है }\end{array}$ & 1 & 2 & 3 & 4 & 5 & 9 \\
\hline $\begin{array}{r}\text { Child requires Vitamin } D \\
\text { for proper formation of } \\
\text { bones }\end{array}$ & $\begin{array}{l}\text { हड्डियों के सही गठन के लिए बच्चे } \\
\text { मे विटामिन डी आवश्यक है }\end{array}$ & 1 & 2 & 3 & 4 & 5 & 9 \\
\hline $\begin{array}{r}\text { Child requires Iron for } \\
\text { blood development }\end{array}$ & $\begin{array}{l}\text { रक्त विकास के लिए आयरन बच्चे } \\
\text { मे आवश्यक है }\end{array}$ & 1 & 2 & 3 & 4 & 5 & 9 \\
\hline $\begin{array}{r}\text { Vitamin A helps in } \\
\text { formation of healthy skin } \\
\text { and hair of child }\end{array}$ & $\begin{array}{l}\text { बच्चे की स्वस्थ त्वचा और बालो के } \\
\text { गठन मे विटामिन ए मदद करता है }\end{array}$ & 1 & 2 & 3 & 4 & 5 & 9 \\
\hline $\begin{array}{r}\text { Vitamin A is good for } \\
\text { proper eyesight of child }\end{array}$ & $\begin{array}{c}\text { बच्चे की सही दृष्टि के लिए } \\
\text { विटामिन ए अच्छा है }\end{array}$ & 1 & 2 & 3 & 4 & 5 & 9 \\
\hline $\begin{array}{r}\text { Child requires all nutrients } \\
\text { for proper growth and } \\
\text { development }\end{array}$ & $\begin{array}{l}\text { बच्चे को सही विकास और वृद्धि के } \\
\text { लिए सभी पोषको की जरूरत है }\end{array}$ & 1 & 2 & 3 & 4 & 5 & 9 \\
\hline $\begin{array}{r}\text { The food should have } \\
\text { lodine which is essential } \\
\text { for child }\end{array}$ & $\begin{array}{l}\text { खाने मे आयोडिन होना चाहिए जो } \\
\text { कि बच्चे के लिए महत्वपूर्ण है }\end{array}$ & 1 & 2 & 3 & 4 & 5 & 9 \\
\hline $\begin{array}{r}\text { Fat content in food is } \\
\text { required for physical } \\
\text { growth of child }\end{array}$ & $\begin{array}{l}\text { बच्चे के शारीरिक विकास के लिए } \\
\text { खाने मे युक्त फैट आवश्यक है }\end{array}$ & 1 & 2 & 3 & 4 & 5 & 9 \\
\hline $\begin{array}{r}\text { The food should be easy } \\
\text { to digest }\end{array}$ & $\begin{array}{r}\text { खाना पचाने के लिए आसान होना } \\
\text { चाहिए }\end{array}$ & 1 & 2 & 3 & 4 & 5 & 9 \\
\hline $\begin{array}{l}\text { There should be variety in } \\
\text { food given to child so that } \\
\text { all nutrients are provided }\end{array}$ & $\begin{array}{l}\text { दिए गए आहार मे विविधता होनी } \\
\text { चाहिए ताकि सभी पोषक प्रदान किए } \\
\text { जा सके }\end{array}$ & 1 & 2 & 3 & 4 & 5 & 9 \\
\hline $\begin{array}{r}\text { Zinc is important for } \\
\text { formation of protein in } \\
\text { body which helps in growth } \\
\text { of child }\end{array}$ & $\begin{array}{l}\text { जिंक शरीर मे प्रोटीन की बनावट के } \\
\text { लिए महत्वपूर्ण है जो बच्चे के } \\
\text { विकास मे सहायक है }\end{array}$ & 1 & 2 & 3 & 4 & 5 & 9 \\
\hline $\begin{array}{r}\begin{array}{r}\text { Child requires Zinc for } \\
\text { blood formation }\end{array} \\
\end{array}$ & $\begin{array}{l}\text { रक्त के बनने के लिए बच्चे को } \\
\text { जिंक की जरूरत होती है }\end{array}$ & 1 & 2 & 3 & 4 & 5 & 9 \\
\hline $\begin{array}{r}\text { Child requires Zinc to help } \\
\text { maintain a healthy immune } \\
\text { system }\end{array}$ & $\begin{array}{l}\text { एक स्वस्थ प्रतिरक्षा प्रणाली बनाए } \\
\text { रखने के मदद के लिए बच्चे को } \\
\text { जिंक की जरूरत होती है }\end{array}$ & 1 & 2 & 3 & 4 & 5 & 9 \\
\hline
\end{tabular}


Q508 We would like to understand what you think about infant cereals as food for your child. I will now read a list of statements and ask you if you agree with them or not. SINGLE CODING PER STATEMENT

हम समझना चाहेंगें आपके बच्चे के लिए खाद्य के रूप मे आप शिशु सीरियल के बारे मे क्या सोचते है। मै वाक्यों की एक सुची पढूंगा और आपसे उनके साथ सहमति के लिए पूछूंगा ।

\begin{tabular}{|c|c|c|c|c|c|}
\hline $\begin{array}{l}\text { TICK } \\
\text { START }\end{array}$ & & & 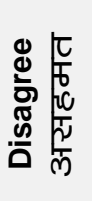 & 离 & 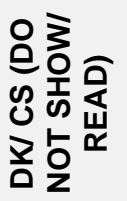 \\
\hline & $\begin{array}{r}\text { A child that is thin or frequently sick } \\
\text { should eat more cereals }\end{array}$ & $\begin{array}{l}\text { एक बच्चा जो कि पतला है या ज्यादा } \\
\text { बीमार होता है उसे ज्यादा अनाज } \\
\text { चाहिए }\end{array}$ & 1 & 2 & 9 \\
\hline & $\begin{array}{r}\text { Cereals are better for my child than } \\
\text { Fast Food }\end{array}$ & $\begin{array}{r}\text { अनाज मेरे बच्चे के लिए फास्ट फूड } \\
\text { से बेहतर है }\end{array}$ & 1 & 2 & 9 \\
\hline & $\begin{array}{r}\text { Homemade cereals are better for my } \\
\text { child than packaged infant cereals }\end{array}$ & $\begin{array}{l}\text { घर पर बने अनाज मेरे बच्चे के लिए } \\
\text { पैकेज्ड अनाज से ज्यादा बेहतर है }\end{array}$ & 1 & 2 & 9 \\
\hline & $\begin{array}{r}\text { Cereals contain much of the energy a } \\
\text { child needs }\end{array}$ & $\begin{array}{l}\text { अनाज मे एक बच्चे के लिए आवश्यक } \\
\text { ज्यादा उर्जा होती है }\end{array}$ & 1 & 2 & 9 \\
\hline & $\begin{array}{l}\text { Cereals contain nutrients that are } \\
\text { lacking in other foods of children's diet }\end{array}$ & $\begin{array}{l}\text { अनाज पोषक युक्त होता है जो कि } \\
\text { बच्चे के अन्य आहार मे कम होते है }\end{array}$ & 1 & 2 & 9 \\
\hline & $\begin{array}{r}\text { Cereals best suited for small children } \\
(6-23 \mathrm{~m}) \text { is different from cereals suited } \\
\text { for children over } 3 \text { years of age and } \\
\text { adults }\end{array}$ & $\begin{array}{l}3 \text { वर्ष की आयु और बडे बच्चो के } \\
\text { लिए उपयुक्त अनाज छोटे बच्चो के } \\
\text { लिए उपयुक्त अनाज से अलग है }\end{array}$ & 1 & 2 & 9 \\
\hline & $\begin{array}{r}\text { Cereals are required for growing } \\
\text { children }\end{array}$ & बढते बच्चे के लिए अनाज जरूरी है & 1 & 2 & 9 \\
\hline & $\begin{array}{r}\text { Cereals are required when only milk is } \\
\text { not sufficient for child }\end{array}$ & $\begin{array}{l}\text { बच्चे के लिए जब केवल दूध प्याप्त } \\
\text { नही है तब अनाज जरूरी है }\end{array}$ & 1 & 2 & 9 \\
\hline & Cereals are filling & अनाज पेट भरने वाले है & 1 & 2 & 9 \\
\hline
\end{tabular}

\section{THANK \& CLOSE THE INTERVIEW}

